\title{
Consistent cost sharing
}

\author{
Maurice Koster
}

Received: 18 March 2011 / Accepted: 3 October 2011 / Published online: 23 November 2011 C) The Author(s) 2011. This article is published with open access at Springerlink.com

\begin{abstract}
A new concept of consistency for cost sharing solutions is discussed, analyzed, and related to the homonymous and natural property within the rationing context. Main result is that the isomorphism in Moulin and Shenker ( $\mathrm{J}$ Econ Theory 64:178-201, 1994) pairs each additive and consistent single-valued mechanism with a corresponding monotonic and consistent rationing method. Then this answers the open question in Moulin (Econometrica 68:643-684, 2000; Handbook of social choice and welfare. Handbooks in economics, pp 289-357, 2002) whether such notion for cost sharing exists. The conclusion is that renown solutions like the average and serial cost sharing mechanisms are consistent, whereas the Shapley-Shubik mechanism is not. Average cost sharing is the only strongly consistent element in this class. The two subclasses of incremental and parametric cost sharing mechanisms are further analyzed as refinement of the main result.
\end{abstract}

Keywords Resource allocation · Cost sharing · Mechanism ·

(Parametric)rationing · Additivity

\section{Introduction}

A group of agents $S$ have demands $q=\left(q_{i}\right)_{i \in S}$ for a single perfectly divisible output and corresponding production costs $C\left(\sum_{i \in S} q_{i}\right)$ have to be shared. A solution is a device that relates each such cost sharing problem to a vector $x$ of individual cost shares such that $\sum_{i \in S} x_{i}=C\left(\sum_{i \in S} q_{i}\right)$. In this paper we will scrutinize the solutions for consistency, a property that is credited a fundamental role in decision problems in various fields of economics and social choice theory—see, e.g. Thomson (2006) and

M. Koster $(\varangle)$

Department of Quantitative Economics, Amsterdam School of Economics,

University of Amsterdam, Amsterdam, The Netherlands

e-mail: mkoster@uva.nl 
Maschler (1990). The idea in the cost sharing context is the following: a subgroup $M$ of agents in $S$ agree to the solution at hand, and 'leave' the cost sharing problem with paying their share of the production cost. The solution is consistent if the remaining agents in $S \backslash M$ can just apply the same device in the remaining or reduced cost sharing problem without altering their individual cost shares. Following Young (1985, p. 19), consistency is a self-similarity property which envisions an idea of fairness of solutions at all levels of cooperation, for any subgroup of agents, according to which 'no subgroup should want to "re-contract".

Where for some models in the resource allocation literature the concept of consistency is transparent and unambiguous (like in rationing, see Thomson 2001; Moulin 2002), for the cost sharing problem it is as ambiguous as the underpinning idea of reduction. It may be seen self-evident to determine the reduced demand profile by $q^{*}=q_{S \backslash M}$, but it is not at all straightforward how to determine the corresponding reduced cost function $C^{*}$. Moulin (2000) questions whether a concept for the cost sharing model exists which is close to the spirit of consistency in the rationing framework. This paper provides an affirmative answer. In particular, it contributes to the literature by the way the ambiguity of choosing an appropriate reduction is by-passed. When it is not clear which 'reasonable' reduction suits a consistent solution best, the proposal is to keep all of them. In turn a solution $\mu$ is consistent if for any cost sharing problem $(q, C)$ under consideration a suitable reduction $\left(q_{S \backslash M}, C^{*}\right)$ exists for each set $M \subset S$ under which the solution is self-similar, i.e. $\mu_{S \backslash M}(q, C)=\mu\left(q_{S \backslash M}, C^{*}\right)$. Under such solution the agents may rationalize the old proposal of cost shares by choosing an appropriate new reduced cost sharing problem. In absence of such reduction, the implementation of the cost sharing solution is problematic if agents hold different opinions to which problem the solution must be applied.

\subsection{Relation to the literature}

This paper fits in the literature on resource allocation and in particular the axiomatic cost sharing literature which discusses structural and characterizing properties of solutions; see e.g. Thomson (2001), Moulin (2002), and Koster (2009b) for general overviews. The proposed property of consistency in this paper is different from that in Sobolev (1973) and Davis and Maschler (1965), where the residual cost is considered as the difference between total cost and remaining cost after the leaving coalition has paid her share. Examples of this approach can be found in Moulin and Shenker (1994), Kolpin (1994), Tijs and Koster (1998), and Albizuri and Zarzuelo (2007). This literature does not provide a concise discussion of consistency as a general property; the presented ideas mainly serve the goal of characterizing solutions under consideration and have therefore a limited say. This paper is closer to the literature following Hart and Mas-Colell (1989). Here the residual cost is considered as the total cost minus the total amount that the remaining agents would have to pay under the cost sharing solution, where potential revision of the demands of the remaining agents is incorporated. Sudhölter (1998) explores Hart and Mas-Colell type of consistency for different cooperative cost games, but which choice of cost game models the situation best is not clear. Leroux (2007) advances by a more profound definition which respects the rich 
cost structure, but turns a homogeneous model into a heterogeneous one. This paper tries to overcome some of the listed shortcomings.

\subsection{Overview of the paper and summary of the results}

Section 2 provides the basic model of homogeneous cost sharing, as well as the notion of a solution in these contexts. The class of incremental mechanisms is introduced. Section 3 introduces the idea of reduced cost sharing problem and defines the concept of consistency for general solutions. Section 4 focuses on the special meaning of consistency for the class of mechanisms with the properties of additivity and constant returns, the class which is known to be isomorphic to the class of monotonic rationing methods (see Moulin and Shenker 1994). Main result is Theorem 4.2, which points out that this isomorphism also pairs the consistent additive mechanisms with the consistent and monotonic rationing methods. Conclusion is that the serial- and average cost sharing mechanism are consistent, and the Shapley-Shubik mechanism is not. Theorem 4.5 pins down the average cost sharing mechanism as the unique additive mechanism that is strongly consistent, i.e. consistent with respect to all eligible reductions. Then these notions of consistency sharply divide the three most popular (additive) cost sharing solutions in the literature. The remaining subsections show how results from the rationing literature may be invoked to obtain further refinements of Theorem 4.2. Two classes are discussed, where we find the discussed renown consistent mechanisms in the intersection. Section 4.2 discusses the class of consistent incremental mechanisms and a characterization. Section 4.3 introduces the parametric cost sharing mechanisms as the cost sharing counterpart of parametric rationing. These mechanisms are characterized using Young (1987) characterization of parametric rationing methods. Finally, Sect. 4.4 discusses the possibility of extending an additive mechanism defined on 2-agent problems to a consistent mechanism; it turns out that there is only one way of doing this.

\section{The model and preliminaries}

Consider a production facility for some perfectly divisible good $Y$, of which the technology is summarized by a cost function $C: \mathbb{R}_{+} \rightarrow \mathbb{R}_{+} ; C(y)$ denotes the minimal (monetary) input to generate $y$ units of $Y$. It will be assumed that $C$ is nondecreasing and there are no fixed costs, so that $C(0)=0$. In addition we shall assume absolutely continuous cost functions. ${ }^{1}$ This technical condition implies that a cost function is differentiable almost everywhere with respect to the Lebesgue measure $\lambda$. By $C^{\prime}$ we denote the marginal cost function, i.e., the function that coincides with the derivative of $C$ whenever the latter exists, and assumes the value 0 otherwise. In particular, $C^{\prime}$ is is $\lambda$-integrable and costs for output level $y$ may be expressed as $C(y)=\int_{0}^{y} C^{\prime}(t) d t .^{2}$

\footnotetext{
${ }^{1}$ For such functions it holds that for all intervals $[a, b] \subset \mathbb{R}_{+}$and $\varepsilon>0$ there is a $\delta>0$ such that for every finite collection of pairwise disjoint intervals $\left(a_{k}, b_{k}\right) \subset[a, b], k=1,2, \ldots, n$ with $\sum_{k=1}^{n}\left(b_{k}-a_{k}\right)<\delta$, we have $\sum_{k=1}^{n}\left|f\left(b_{k}\right)-f\left(a_{k}\right)\right|<\varepsilon$.

2 This follows by the Fundamental Theorem in Lebesgue (1904).
} 
The set of all cost functions is denoted by $\mathcal{C}$. Special cost functions are the base functions $B_{t}$ defined for $t \geq 0$ by $B_{t}(y)=\min \{t, y\}$.

Denote by $\mathcal{N}$ the class of nonempty and finite subsets of the natural numbers, by which we indicate the possible sets of agents in a cost sharing problem; throughout the paper we take the set of agents to be $S \in \mathcal{N}, S=\{1,2, \ldots,|S|\}$. A cost sharing problem for $S$ is an ordered pair $P=(q, C) \in \mathbb{R}_{+}^{S} \times \mathcal{C}$. The interpretation of $P$ is that the agents in $S$ jointly own the production facility, and $q=\left(q_{i}\right)_{i \in S}$ summarizes the individual demands of the agents for $\operatorname{good} Y$; then $q(S)=\sum_{i \in S} q_{i}$ is produced and cost $C(q(S))$ has to be shared. The set of all cost sharing problems for $S$ is denoted $\mathcal{P}^{S}$, and put $\mathcal{P} \equiv \bigcup_{S \in \mathcal{N}} \mathcal{P}^{S}$.

For any $y \in \mathbb{R}_{+}^{S}$ and $M \subseteq S$ the component $y_{S \backslash M} \in \mathbb{R}_{+}^{S \backslash M}$ will also be denoted by $y_{-M}$, or if $M=\{i\}$, by $y_{-i}$. An element $y \in \mathbb{R}_{+}^{S}$ is called vector of cost shares for $(q, c) \in \mathcal{P}^{S}$ if $y(S)=C(q(S))$.

A solution assigns to each cost sharing problem a set of cost shares. For instance, the set of all vectors of cost shares is a solution, as well as the core defined by

$$
\operatorname{CORE}(q, C)=\left\{x \in \mathbb{R}_{+}^{S} \mid x(S)=C(q(S)), x(T) \leq C(q(T)), \text { for all } T \subset S\right\} .
$$

In this paper we will restrict our attention to single-valued solutions, which are called mechanisms. Define $\Delta(S)=\left\{y \in \mathbb{R}_{+}^{S} \mid y(S)=1\right\}$. A mechanism $\mu$ is called an incremental mechanism if for each $q \in \mathbb{R}_{+}^{S}$ there are $k \in \mathbb{N}$ and vectors $\alpha_{1}^{S}, \alpha_{2}^{S}, \ldots, \alpha_{k}^{S} \in$ $\Delta(S), x^{S} \in \mathbb{R}_{+}^{k+1}$ such that

$$
\mu(q, C)=\sum_{\ell=1}^{k} \alpha_{\ell}^{S}\left(C\left(x_{\ell}^{S}\right)-C\left(x_{\ell-1}^{S}\right)\right) \text { for all } C \in \mathcal{C}
$$

and where $0=x_{0}^{S} \leq x_{1}^{S} \leq \ldots \leq x_{k}^{S}=q(S)$. The incremental mechanisms generalize the incemental methods and random order mechanisms as in Weber (1988). Denote the set of all mechanisms by $\mathcal{M}$. Then the set $\mathcal{M}^{I}$ of incremental mechanisms is included in $\mathcal{M}$. For $\mathcal{M}^{*} \subseteq \mathcal{M}$ the mechanisms in $\mathcal{M}^{*}$ with the properties $P_{1}, P_{2}, \ldots, P_{m}$ is denoted $\mathcal{M}^{*}\left(P_{1}, P_{2}, \ldots, P_{m}\right)$.

\subsection{Examples of cost sharing mechanisms}

- The average mechanism $\mu^{\mathrm{AV}}$ is an incremental mechanism with

$$
k=1, x_{k}^{S}=q(S), \alpha_{k}^{S}=\frac{q}{q(S)} \quad \text { whenever } q(S)>0
$$

- Denote the permutations of $S$ by $\Pi(S)$. For $q \in \mathbb{R}_{+}^{S}$ there is an ordering permutation $\sigma \in \Pi(S)$ such that $q_{\sigma(i)} \leq q_{\sigma(j)} \Leftrightarrow \sigma(i) \leq \sigma(j)$ for all $i, j \in S$. Then take $k=|S|$ and 


$$
\begin{gathered}
x_{\ell}^{S}=\sum_{p \leq \sigma^{-1}(\ell)-1} q_{\sigma(p)}+(|S|-\ell+1) q_{\sigma(\ell)} \text { and } \\
\alpha_{\ell i}^{S}= \begin{cases}1 /(|S|-\ell+1) & \text { if } \sigma(i) \geq \ell, \\
0 & \text { else. }\end{cases}
\end{gathered}
$$

The corresponding incremental mechanism is the serial mechanism (Moulin and Shenker 1992) which is denoted $\mu^{\mathrm{SR}}$.

- Let $\sigma: \mathbb{N} \rightarrow \mathbb{N}$ be a bijection. Take $\pi \in \Pi(S)$ that preserves the ordering of agents by $\sigma$, i.e., such that $\pi(i) \leq \pi(j) \Leftrightarrow \sigma(i) \leq \sigma(j)$. The marginal mechanism $\mu^{\sigma}$ is defined on $\mathcal{P}^{S}$ by the choice

$$
x_{\ell}^{S}=\sum_{p \in S ; p \leq \pi^{-1}(\ell)} q_{\pi(p)} \quad \text { and } \quad \alpha_{\ell i}^{S}=1 \Longleftrightarrow \pi(i)=\ell
$$

Then $\mu^{\sigma}(P)$ is the marginal vector with respect to the induced stand alone cost game (see Young 1985, 1994). If no confusion arises it will be convenient to express $\mu^{\sigma}(P)$ as $\mu^{\pi}(P)$.

- The Shapley-Shubik mechanism $\mu^{\mathrm{SS}}$ (Shubik 1962) calculates the Shapley value for the stand alone cost game for each cost sharing problem $P=(q, C) \in \mathcal{P}^{S}$ :

$$
\mu^{\mathrm{SS}}(P)=\frac{1}{|S| !} \sum_{\pi \in \Pi(S)} \mu^{\pi}(P)
$$

This mechanism is incremental for $x_{\ell}^{S}$ 's generated by the pooled demands $\{q(U) \mid U \subseteq S\}$ and

$$
\alpha_{\ell i}^{S}=1 /|S| ! \cdot \mid\left\{\pi \in \Pi(S) \mid\left[x_{\ell-1}^{S}, x_{\ell}^{S}\right] \subseteq\left[\sum_{j ; \pi^{-1}(j)<i} q_{j}, \sum_{j ; \pi^{-1}(j) \leq i} q_{j}\right]\right\} .
$$

Additional notation $\mathbb{I}_{X}: \mathbb{R} \rightarrow\{0,1\}$ is the indicator function with respect to $X \subset \mathbb{R}$, such that $\mathbb{I}_{X}(t)=1 \Leftrightarrow t \in X$.

\section{Consistency}

Consider the cost sharing problem $P=(q, C)$ for $S=\{1,2,3\}$ where $C(y)=y^{2}$ and $q=(1,2,3)$. One way to look at $P$ is that not only costs $C(6)=36$ have to be distributed, but also the total of instantaneous production of 6 units. Suppose that agent 2 is allocated 2 units in total, represented through the production levels in some (measurable) set $Y \subset[0,6]$. Then $Y$ agrees with the cost share $\mu_{2}(P)$ if only the aggregate marginal costs corresponding to the allocated units equals the cost share of agent 2 , i.e., $\int_{Y} C^{\prime}(t) \mathrm{d} t=\mu_{2}(P)$. In other words, a unit $y \in[0,6]$ is considered to have an impact $C^{\prime}(y)$ on total cost and the aggregate impact of agent 2 for receiving the units in 
$Y$ is given by $\int_{Y} C^{\prime}(t) \mathrm{d} t$ - the ultimate liability of agent 2 in $P$. For example, assume average cost sharing so that the cost shares in $P$ are given by $\mu^{\mathrm{AV}}(P)=(6,12,18)$. Then the set $Y=[2,4]$ not only suffices the need for production of agent 2 , but agrees with her cost share as well since $\int_{Y} C^{\prime}(t) \mathrm{d} t=12=\mu_{2}^{\mathrm{AV}}(P)$. In a similar fashion the sets

$$
X=\left[0, \frac{1}{2}\right) \cup\left[\frac{11}{2}, 6\right] \text { and } Z=\left[\frac{1}{2}, 2\right) \cup\left(4, \frac{11}{2}\right)
$$

match the demand of agents 1 and 3 with their respective cost shares. Note that $(X, Y, Z)$ is a partition of $[0,6]$.

Now assume agent 2 leaves the cost sharing problem, taking with her the set of units $Y$ and leaving $\mu_{2}^{\mathrm{AV}}(P)$ as contribution to the total cost of production. The reduced cost function summarizes the cost structure of all production levels except for those that are paid for by agent 2, i.e., the truncation of $C$ over $Y$ defined by

$$
C_{Y}(y)= \begin{cases}C(y) & \text { if } y \leq 2 \\ C(y+2)-12 & \text { if } y>2\end{cases}
$$

There is no unique way according to define allocations of production levels matching the cost shares. For example, another possibility is the partition of $[0,6]$ in

$$
X=\left[\frac{5}{2}, \frac{7}{2}\right], Y=[0,1] \cup[5,6], Z=\left(1, \frac{5}{2}\right) \cup\left(\frac{7}{2}, 5\right)
$$

In this case the reduced cost function resulting from truncation of $C$ over $Y$ defined by

$$
C_{Y}(y)= \begin{cases}C(y+1)-C(1) & \text { if } y \leq 4 \\ C(y+2)-12 & \text { if } y>4\end{cases}
$$

This idea of matching production levels to cost shares can be extended. Assume that the produced units are handed out instantaneously, such that (a) agent 2 is assigned a share $\varphi_{2}(t)$ of each produced unit $t \in[0,6]$, and, moreover, (b) that she contributes $\varphi_{2}(t) C^{\prime}(t)$ to the cost $C^{\prime}(t)$ of this unit. Then, similar to the above examples, $\varphi$ agrees with both agent 2's demand and cost share if the total allocation of units equals $\int_{0}^{6} \varphi_{2}(t) \mathrm{d} t=2=q_{2}$ and the aggregate contribution toward total costs equals $\int_{0}^{6} \varphi_{2}(t) C^{\prime}(t) \mathrm{d} t=12=\mu_{2}^{\mathrm{AV}}(P)$.

Notice that the vector function $\varphi(t)=\left(\frac{1}{6}, \frac{1}{3}, \frac{1}{2}\right)$ defines a production device according to which for each agent the allocated units match the cost share simultaneously. This function may be used to define the reduced cost function $C_{\varphi,-2}$ corresponding to a reduction by agent 2 . First of all, note that $\Phi(y):=\int_{0}^{y} \varphi_{2}(t) \mathrm{d} t$ units of the first $y$ production levels are handed out to agent 2 , for which she pays $\int_{0}^{y} \varphi(t) C^{\prime}(t) \mathrm{d} t$. Hence the remaining cost in $y-\Phi(y)$ is $C_{\varphi,-2}(y-\Phi(y))=C_{\varphi,-2}\left(\frac{2}{3} y\right)$ which equals the total cost of producing $y$ units minus the contribution of agent 2 , that is 


$$
C_{\varphi,-2}\left(\frac{2}{3} y\right)=C(y)-\int_{0}^{y} \varphi_{2}(t) C^{\prime}(t) \mathrm{d} t=\frac{2}{3} y^{2} \text { for all } y \in[0,6]
$$

or $C_{\varphi,-2}(y)=\frac{3}{2} y^{2}$ for $y \in[0,4]$. If we now define $C_{\varphi,-2}(y)=C(y+2)-\mu_{2}^{\mathrm{AV}}(P)$ for $y>4$, then $\left(q\{1,3\}, C_{\varphi,-2}\right)$ is also considered as reduction of $P$ by agent 2 .

\subsection{Reduction and consistency, formal definitions}

Suppose that for $P=(q, C) \in \mathcal{P}^{S}$ and mechanism $\mu$ there is a mapping $\varphi$ : $[0, q(S)] \rightarrow \Delta(S)$ with the properties that for all $i \in S$

$$
\begin{aligned}
& \int_{0}^{q(S)} \varphi_{i}(t) \mathrm{d} t=q_{i}, \\
& \int_{0}^{q(S)} \varphi_{i}(t) C^{\prime}(t) \mathrm{d} t=\mu_{i}(q, C) .
\end{aligned}
$$

Basically this means that $\varphi_{i}$ can be taken to 'represent' agent $i$ 's demand and cost share: unit $t$ is produced and agent $i$ gets the fraction $\varphi_{i}(t)$ of it at the price $\varphi_{i}(t) C^{\prime}(t)$. Then condition (2) is no more than translating that the total of allocated (partial) units sum up to the agent's demand, and (3) ascertains that the corresponding marginal costs are in line with the proposed solution. Such mapping $\varphi$ which matches demands and cost shares for all agents simultaneously is called a reduction map for $(\mu, P)$. If a reduction map for $(\mu, P)$ exists then $P$ is called reducible with respect to $\mu$.

Importantly, recall that $\varphi$ sees to a disjoint allocation of units, just to ensure that after an agent leaves the cost sharing problem, the remaining group of agents do not need to bargain over the same units (see Lemma 5.3 ). ${ }^{3}$

Note that we allow for $\varphi$ to depend on the cost sharing problem at hand. Below we will discuss an important class of solutions where $\varphi$ can be taken irrespective of the cost sharing problem, like in the following example.

Example 3.1 For any cost sharing problem $P=(q, C), \varphi(t)=q / q(S)$ defines a reduction map for $\left(\mu^{\mathrm{AV}}, P\right)$.

Take a reduction map $\varphi$ for $(\mu, P), i \in S$. Let $\Phi_{i}$ be the primitive of $\varphi_{i}$ such that $\Phi_{i}(0)=0$. Then $\varphi$ defines a reduced cost sharing problem for agent $i, P_{\varphi,-i}=$ $\left(q_{-i}, C_{\varphi,-i}\right) \in \mathcal{P}^{S \backslash\{i\}}$, where $C_{\varphi,-i} \in \mathcal{C}$ is implicitly defined on $[0, q(S \backslash\{i\})]$ by

$$
C_{\varphi,-i}\left(y-\Phi_{i}(y)\right)=\int_{0}^{y}\left(1-\varphi_{i}(t)\right) C^{\prime}(t) \mathrm{d} t \text { for } y \in[0, q(S)],
$$

\footnotetext{
3 I thank Justin Leroux for making this point.
} 


$$
C_{\varphi,-i}(y)=C\left(y+q_{i}\right)-\int_{0}^{q(S)} \varphi_{i}(t) C^{\prime}(t) \mathrm{d} t \text { for } y>q(S \backslash\{i\}) .
$$

The second line says that, beyond production levels $q(S)$, the original cost function is truncated by the cost paid by agent $i .{ }^{4}$ Moreover, note that $C_{\varphi,-i}$ is non-decreasing as well. ${ }^{5}$ If the inverse $g$ of the mapping $y \mapsto y-\Phi_{i}(y)$ exists, then $C_{\varphi,-i}$ is explicitly given by

$$
C_{\varphi,-i}(y)=\int_{0}^{g(y)}\left(1-\varphi_{i}(t)\right) C^{\prime}(t) \mathrm{d} t
$$

As we have seen before reductions are in general not unique; in fact, each cost sharing problem can be associated uncountably many reductions. Then a concept of consistency would be too restricted if it were confined to just one of these. To avoid the ambiguity of choosing the proper reduction, $\mu$ will be called consistent if the restriction of the vector of cost shares in the original situation is available for some reduced cost sharing problem. This guarantees that the remaining agents can at least choose a reformulation of the cost sharing problem which preserves the status quo solution. If the status quo solution is stable irrespective of the choice of reduction, then the solution is strongly consistent. Formally:

Consistency (CONS) For all $P \in \mathcal{P}^{S}$, there exists a reduction map $\varphi$ for $(\mu, P)$ such that $\mu_{-i}(P)=\mu\left(P_{\varphi,-i}\right)$ for all $i \in S$.

Strong Consistency (SCONS) $\mu$ is consistent and for all $P \in \mathcal{P}^{S}$, and all reduction mappings $\varphi$ for $(\mu, P), \mu_{-i}(P)=\mu\left(P_{\varphi,-i}\right)$ for all $i \in S$.

Example 3.2 Below we will show that the marginal mechanisms $\mu^{\sigma}$ are all consistent. However, they are not strongly consistent. To see that strong consistency is violated, consider the problem $P=(q, C) \in \mathcal{P}^{\{1,2,3\}}$ with $C(y)=\int_{0}^{y} \mathbb{I}_{[0,1] \cup[2,3]}(t) \mathrm{d} t$ and $q=(1,1,1)$. If $\pi$ is the identity permutation on $\{1,2,3\}, \mu^{\pi}(q, C)=(1,0,1)$. Then $\varphi$ defined by $\varphi(t)=\left(\mathbb{I}_{[2,3]}, \mathbb{I}_{[1,2)}, \mathbb{I}_{[0,1)}\right)$ is a reduction map for $\left(\mu^{\pi}, P\right)$, but $\mu_{2}^{\pi}\left(P_{\varphi,-1}\right)=\mu_{2}^{\pi}\left(q\{2,3\}, C_{\varphi,-1}\right)=1 \neq \mu_{2}^{\pi}(q, C)$.

Example 3.3 Note that the reductions by agent 2 in the introductory example do not affect the cost shares of agents 1 and 3 , as long as $\mu^{\mathrm{AV}}$ is used. No matter the reduction, $\mu^{\mathrm{AV}}$ always calculates the same cost shares for the remaining agents as it is a strongly consistent mechanism. To see this, consider a cost sharing problem $P=(q, C) \in \mathcal{P}^{S}$ for $S \subseteq N$, and assume that $q(S)>0$. Then $P$ is reducible by $t \mapsto q / q(S)$. Now take $i \in S$ and an arbitrary reduction map $\varphi$ for $\left(\mu^{\mathrm{AV}}, P\right)$. Then

\footnotetext{
4 This is to ensure that the reduction is still absolutely continuous; another non-decreasing continuation would serve the exposition just as well.

5 To see this, consider $v, w \in[0, q(S \backslash\{i\})], v<w$. The mapping $y \mapsto y-\Phi_{i}(y)$ is non-decreasing, so for $v=y^{*}-\Phi_{i}\left(y^{*}\right)$ and $w=\tilde{y}-\Phi_{i}(\tilde{y})$, it must hold $y^{*}<\tilde{y}$. Then $C_{\varphi,-i}(w)-C_{\varphi,-i}(v)=$ $\int_{y^{*}}^{\tilde{y}}\left(1-\varphi_{i}(t)\right) C^{\prime}(t) \mathrm{d} t \geq 0$.
} 


$$
\begin{aligned}
\mu^{\mathrm{AV}}\left(P_{\varphi,-i}\right) & =\mu^{\mathrm{AV}}\left(q_{-i}, C_{\varphi,-i}\right)=\frac{q_{-i}}{q(S \backslash\{i\})} C_{\varphi,-i}(q(S \backslash\{i\})) \\
& =\frac{q_{-i}}{q(S \backslash\{i\})}\left\{C(q(S))-\frac{q_{i}}{q(S)} C(q(S))\right\}=\mu_{-i}^{\mathrm{AV}}(P) .
\end{aligned}
$$

Standard in the literature is consistency as a stability notion with respect to reduction by coalitions of agents rather than individuals. The former approach generalizes in a natural way to coalitional consistency, by considering the collective of leaving agents as a single agent. Consider a reduction mapping $\varphi$ for $(\mu, P)$ where $P=(q, C) \in \mathcal{P}^{S}$. The reduced cost sharing problem with respect to $M$ and $\varphi, P_{\varphi,-M}=\left(q_{S \backslash M}, C_{\varphi,-M}\right)$, is defined implicitly and in analogy to 4 by

$$
C_{\varphi,-M}\left(y-\Phi_{M}(y)\right)=\int_{0}^{y}\left(1-\varphi_{M}(t)\right) C^{\prime}(t) \mathrm{d} t,
$$

where $\varphi_{M}:=\sum_{i \in M} \varphi_{i}$, and $\Phi_{M}$ is a primitive of $\varphi_{M}$ such that $\Phi_{M}(0)=0$.

Coalitional Consistency For all $P \in \mathcal{P}^{S}, M \subseteq S$, there exists a reduction mapping $\varphi$ so that $\mu_{-M}(P)=\mu\left(P_{\varphi,-M}\right)$.

Below, it will be convenient to stick to the single-agent interpretation of consistency and there is no loss of generality in doing so:

Lemma 3.4 $\mu$ is consistent iff $\mu$ is coalitionally consistent.

Note that the above concepts require that-given some (strongly) consistent solution-any cost sharing problem is reducible. Then this rules out the equal split mechanism $\mu^{\mathrm{E}}(q, C):=C(q(S)) /|S|$, which is not consistent for the fact that it may not be reducible. ${ }^{6}$

Nevertheless, below it will be argued that reducibility is actually not too much to ask for. As in Tijs and Koster (1998) associate each $P=(q, C) \in \mathcal{P}^{S}$ with the pessimistic cost sharing problem $\left(q, C_{P}^{*}\right) \in \mathcal{P}^{S}$, where the pessimistic cost function $C_{P}^{*}$ is defined by

$$
C_{P}^{*}(y)= \begin{cases}\sup \left\{\int_{T} C^{\prime}(t) \mathrm{d} t \mid T \subseteq[0, q(S)], \lambda(T)=y\right\} & \text { if } y \in[0, q(S)], \\ C(y) & \text { if } y>q(S) .\end{cases}
$$

The function $C_{P}^{*}$ is the decreasing rearrangement ${ }^{7}$ of $C$ that relates each level of aggregate demand $y \in[0, q(S)]$ to a specific upper bound on costs, given by the maximum of corresponding aggregate marginal cost on $[0, q(S)]$. Ryff (1970) implies that $C_{P}^{*}$ is absolutely continuous and that it indeed belongs to $\mathcal{C}$. Moreover, it is easy to show that

\footnotetext{
6 Koster (2009a,b) shows how consistency may be discussed in a framework without the assumption of $\mathrm{CR}$, but it is beyond the scope of this paper. However, under CR the general idea is equivalent to the one under consideration.

7 See Hardy et al. (1959).
} 
$C_{P}^{*}$ is concave on $[0, q(S)]$. In particular, $\operatorname{CORE}^{*}(q, C):=\operatorname{CORE}\left(q, C_{P}^{*}\right) \neq \varnothing$ and in general this set is large. Suppose that a vector of cost shares $y$ is not in $\operatorname{CORE}^{*}(q, C)$. Then there exists a coalition which may object against $y$, as it is supposed to contribute more than can be justified by even the aggregated highest marginal costs. In this sense the pessimistic core provides weaker incentive constraints than the stand alone core (see, e.g. Sharkey 1982; Young 1985, 1994). Lemma 3.5 below shows that consistency can only be a meaningful concept for solutions that are in line with the constraints imposed by the pessimistic core.

\section{Lemma 3.5}

1. If $\mu(P) \in \operatorname{CORE}^{*}(P)$ then $(\mu, P)$ is reducible.

2. If $\mu$ is consistent, then $\mu(P) \in \operatorname{CORE}^{*}(P)$ for all $P \in \mathcal{P}$.

Note that this does not mean a full characterization of the consistent solutions; consistency by definition implies reducibility, but not the other way around. To see this, consider the mechanism $\mu$ defined by

$$
\mu(P)= \begin{cases}\mu^{\mathrm{AV}}(P) & \text { for } P \in \mathcal{P}^{S},|S|>2 \\ \mu^{\mathrm{SR}}(P) & \text { otherwise. }\end{cases}
$$

The mechanism so defined is reducible for any cost sharing problem $P=(q, c) \in \mathcal{P}^{S}$ with $|S|>2$ (see Example 3.1). But a reduction from a 3-agent problem to a 2-agent problem will change the cost shares for the remaining agents.

\section{Characterizing consistent and additive cost sharing}

The two main characterizing properties in the state of the art cost sharing literature are additivity and constant returns. Additivity is propagated as an accounting convention, allowing for the decomposition of a cost sharing problem in several cost components without altering the final cost allocations. Constant returns declares price of the good equal to marginal costs in case of linear cost functions. Formally, for mechanisms $\mu$ we define:

Additivity $(\mathrm{ADD}) \mu\left(q, C_{1}+C_{2}\right)=\mu\left(q, C_{1}\right)+\mu\left(q, C_{2}\right)$ for all demand profiles $q$ and $C_{1}, C_{2} \in \mathcal{C}$.

Constant Returns $(\mathrm{CR}) \mu\left(q, C_{\vartheta}\right)=\vartheta q$ for $C_{\vartheta} \in \mathcal{C}$ given by $C_{\vartheta}(y)=\vartheta y$ for some $\vartheta \in \mathbb{R}_{+}$.

Note that $\mu^{\mathrm{AV}}, \mu^{\mathrm{SR}}, \mu^{\mathrm{SS}}$ belong to $\mathcal{M}(\mathrm{ADD}, \mathrm{CR})$, the class of all mechanisms with both properties ADD and CR. ${ }^{8}$ Obviously, $\mu^{\mathrm{E}}$ satisfies ADD but not CR.

We will now focus on the result of Moulin and Shenker (1994) which shows the close connection between the elements of $\mathcal{M}(\mathrm{ADD}, \mathrm{CR})$ and rationing methods. First we will provide the necessary rationing terminology.

\footnotetext{
8 An excellent overview on additive cost sharing in the homogeneous model is Moulin (2002); non-additive mechanisms are proposed and analyzed by, e.g., Tijs and Koster (1998), Koster (2002), and Hougaard and Thorlund-Petersen (2001).
} 
A rationing problem for agents in $S \subseteq N$ consists of a pair $(q, t) \in \mathbb{R}_{+}^{S} \times \mathbb{R}_{+}$ such that $q(S):=\sum_{i \in S} q_{i} \geq t$. Here $q$ is usually interpreted as a vector of claims, and $t$ the amount to be divided. A rationing method $r$ associates to any rationing problem $(q, t) \in \mathbb{R}_{+}^{S} \times \mathbb{R}_{+}$a vector $r(q, t) \in \mathbb{R}_{+}^{S}$ such that $r_{i}(q, t) \leq q_{i}$ for all $i \in S$ and $\sum_{i \in S} r_{i}(q, t)=t$. So, nobody is granted more than her claim while the available amount is fully distributed. Then $r$ is monotonic whenever $t \leq t^{\prime}$ implies $r(q, t) \leq r\left(q, t^{\prime}\right)$ for all $t, t^{\prime}, q \in \mathbb{R}_{+}^{S}$. Then each such rationing method defines for all $q \in \mathbb{R}_{+}^{S}$ a monotonic (and continuous) path $t \mapsto r(q, t)$ from 0 to $q$. Denote the set of all monotonic rationing methods by $\mathcal{R}$. We have the following:

Theorem 4.1 (Moulin and Shenker 1994) Define the mappings

$$
\begin{aligned}
& r \rightarrow \mu: \mu(q, C)=\int_{0}^{q(S)} C^{\prime}(t) \mathrm{d} r(q, t) \text { for all } C \in \mathcal{C}, q \in \mathbb{R}_{+}^{S}, \\
& \mu \rightarrow r: r(q, t)=\mu\left(q, B_{t}\right) \text { for all }(q, t) \in \mathbb{R}_{+}^{S} \times \mathbb{R}_{+} .
\end{aligned}
$$

These define a linear isomorphism from $\mathcal{R}$ into $\mathcal{M}(\mathrm{ADD}, \mathrm{CR})$ and back.

This theorem, formulated as in Moulin (2002), shows that each mechanism $\mu \in$ $\mathcal{M}(\mathrm{ADD}, \mathrm{CR})$ is fully characterized through its rationing method $r$; I shall consequently write $\mu=\mu^{r}$. For instance, any incremental mechanism $\mu$ as in (1) with the property of CR has a corresponding piecewise linear rationing method $r$ such that

$$
\frac{\partial}{\partial t} r(q, t)=\alpha_{\ell}^{S} \text { for all } t \in\left(x_{\ell-1}^{S}, x_{\ell}^{S}\right)
$$

in case $x_{\ell}^{S} \neq x_{\ell-1}^{S}$. Well-known in the cost sharing literature are the following members of $\mathcal{M}^{I}$ :

- $\mu^{\mathrm{AV}}$ corresponds to the proportional rationing method $r^{\mathrm{P}}$,

$$
r^{\mathrm{P}}(q, t)= \begin{cases}0 & \text { if } q(S)=0, \\ \frac{q}{q(S)} t & \text { otherwise. }\end{cases}
$$

- $\mu^{\mathrm{SR}}$ corresponds to the uniform gains method $r^{\mathrm{UG}}, r_{i}^{\mathrm{UG}}(q, t)=\min \left\{q_{i}, \omega\right\}$, where $\omega$ solves $\sum_{j \in S} \min \left\{q_{j}, \omega\right\}=t$.

- $\mu^{\sigma}$ corresponds to the priority rationing method $r^{\sigma}$ : consider $\pi \in \Pi(S)$ that preserves the ordering of $\mathbb{N}$ by $\sigma$. Let $k$ be the integer such that $\sum_{j=1}^{k} q_{\pi(j)} \leq t \leq$ $\sum_{j=1}^{k+1} q_{\pi(j)}$, then

$$
r_{i}^{\sigma}(q, t)= \begin{cases}q_{i} & \text { for } i=\pi(1), \pi(2), \ldots, \pi(k), \\ t-\sum_{j=1}^{k} q_{\pi(j)} & \text { for } i=\pi(k+1), \\ 0 & \text { otherwise. }\end{cases}
$$

If no confusion arises, we will also write $r^{\pi}$ instead of $r^{\sigma}$. 
- $\mu^{\mathrm{SS}}$ corresponds to the random priority rationing method is defined as the average of all priority rationing methods, i.e., $r^{\mathrm{RP}}(q, t)=\frac{1}{|S| !} \sum_{\pi \in \Pi(S)} r^{\pi}(q, t)$.

Direct consequence from Theorem 4.1 is that mechanisms in $\mathcal{M}(\mathrm{ADD}, \mathrm{CR})$ yield reducible problems always: if $\mu=\mu^{r}$ then $\varphi:[0, q(S)] \rightarrow \Delta(S)$ defined through $\varphi(t)=\frac{\partial}{\partial t} r(q, t)$ is a reduction map for $(\mu, P)$ for any $P$. So we observe two facts: (a) the reduction map is special in the sense that it does not depend on the cost sharing problem at hand, and (b) this makes consistency a non-void concept within $\mathcal{M}$ (ADD, CR). Moreover, for cost sharing problems $(q, C)$ with $C(y)=\alpha y$ for some $\alpha \in \mathbb{R}_{+}$we have, by Lemma 3.5, $\mu(q, C) \subseteq \operatorname{CORE}^{*}(q, C)=\{\alpha q\}$. This shows that under ADD, CONS implies CR.

\subsection{The main results}

Within the rationing context the idea of consistency is transparent and intuitive: a rationing method $r$ is called consistent if for all rationing problems $(q, t)$ among agents in $S, r_{-i}(q, t)=r\left(q_{-i}, t-r_{j}(q, t)\right)$ for all $i \in S .{ }^{9}$ Hence, consistency states that with removing an agent from the cooperative $S$, and taking all the resources that are allocated to this agent, renewed allocation of the remaining pieces within the reduced society does not make a difference as long as $r$ is used. As Moulin (2002) puts it, 'changing the status of an agent from active participant to passive expense of resources does not alter the overall distribution'. Renown examples of consistent rationing methods are $r^{\mathrm{P}}, r^{\mathrm{UG}}, r^{\sigma}$, while $r^{\mathrm{RP}}$ is not consistent - see, e.g., Moulin (2002).

Within the context of Theorem 4.1, consistency smoothly transfers from the cost sharing context to the rationing model and back. The mapping $r \mapsto \mu^{r}$ defines an isomorphism between the consistent elements in $\mathbb{R}$ and $\mathcal{M}(\mathrm{ADD}, \mathrm{CONS})$.

Theorem 4.2 $\mu \in \mathcal{M}$ (ADD, CONS) if and only if $\mu=\mu^{r}$ and $r$ is a consistent and monotonic rationing method.

Using the knowledge about the rationing methods underlying the above mentioned cost sharing mechanisms we obtain:

Corollary 4.3 $\mu^{\mathrm{SR}}, \mu^{\mathrm{AV}}$, and all marginal mechanisms $\mu^{\sigma}$ are consistent, but $\mu^{\mathrm{SS}}$ is not.

Although the idea of reduction map is reminiscent of a rationing method, consistency is not at all confined to additive cost sharing mechanisms only as Theorem 4.2 might suggest. On the contrary, the example below shows how to derive non-additive and consistent mechanisms, by the composition of the pessimistic transformation of a cost sharing problem and a consistent and additive mechanism. In this case the reduction map depends on the cost sharing problem at hand.

Example 4.4 Define the pessimistic marginal mechanism $\mu^{\sigma, *}$ by $\mu^{\sigma, *}(q, C):=$ $\mu^{\sigma}\left(q, C_{P}^{*}\right)$ for $P=(q, C)$. Then clearly $\mu^{\sigma, *}$ is not additive. We will show that

\footnotetext{
9 In fact the notion is usually defined in terms of general sets of agents leaving, but is derived from repeated application of this statement.
} 
it is consistent. Assume that $C$ is concave cost function, so that $\mu^{\sigma, *}(q, C)=$ $\mu^{\sigma}(q, C)$. Define a reduction mapping $\varphi$ through $\varphi_{i}:=\mathbb{I}_{Y(i)}$, where $Y(i)=$ $\left[\sum_{j \in S: \sigma(j)<\sigma(i)} q_{j}, \sum_{j \in S: \sigma(j) \leq \sigma(i)} q_{j}\right]$ for all $i \in S$. Then the reduced cost sharing problem with respect to agent $i$ is given by $\left(q_{-i}, C_{Y(i)}\right)$, and $C_{Y(i)}$ is again a concave function. So

$$
\mu_{-i}^{\sigma, *}(q, C)=\mu_{-i}^{\sigma}(q, C)=\mu^{\sigma}\left(q_{-i}, C_{\varphi,-i}\right)=\mu^{\sigma, *}\left(q_{-i}, C_{\varphi,-i}\right) .
$$

If $C$ is not concave, we may basically apply the same reasoning to its decreasing rearrangement $C_{P}^{*}$. Let $f_{P}:[0, q(S)] \rightarrow[0, q(S)]$ a $\lambda$-measurable mapping implementing the rearrangement $C_{P}^{*}$ such that for all $t \in[0, q(S)],\left(C_{P}^{*}\right)^{\prime}(t)=C^{\prime}\left(f_{P}(t)\right)$. Then a reduction mapping $\varphi$ is defined for $P$ by $\varphi_{i}=\mathbb{I}_{f_{P}(Y(i))}$. By choice of the reduction we obtain $\left(C_{\varphi,-i}\right)_{P}^{*}=\left(C_{f_{P}(Y(i))}\right)_{P}^{*}=\left(C_{P}^{*}\right)_{Y(i)}$. By concavity of $C_{P}^{*}$ we have

$$
\begin{aligned}
\mu_{-i}^{\sigma, *}(q, C) & =\mu_{-i}^{\sigma}\left(q, C_{P}^{*}\right)=\mu^{\sigma}\left(q_{-i},\left(C_{P}^{*}\right)_{Y(i)}\right) \\
& =\mu^{\sigma}\left(q_{-i},\left(C_{\varphi,-i}\right)_{P}^{*}\right)=\mu^{\sigma, *}\left(q_{-i}, C_{\varphi,-i}\right) .
\end{aligned}
$$

Recall that $\mu^{\mathrm{SS}}$ is additive and not consistent. Then the above example can be taken as proof of the logical independence of the properties additivity and consistency.

It can be shown that for non-trivial cost sharing problems $P$-with all positive demands - there are uncountably many reduction maps for $(\mu, P)$ when $\mu \in$ $\mathcal{M}(\mathrm{ADD}, \mathrm{CR})$. In particular, a strongly consistent $\mu$ should be stable with respect to all of these reductions - which turns out to be too demanding for mechanisms using more than only the level of aggregate cost.

Theorem 4.5 $\mu \in \mathcal{M}(\mathrm{ADD}, \mathrm{SCONS})$ if and only if $\mu=\mu^{\mathrm{AV}}$.

\subsection{Characterization of consistent incremental mechanisms}

The most popular cost sharing mechanisms belong to the class of incremental mechanisms $\mathcal{M}^{I}$, which makes the result below worthwhile.

Proposition 4.6 Let $\mu \in \mathcal{M}^{I}$ (ADD, CR) be given by (1). Then $\mu$ satisfies CONS if and only if for all $S, q \in \mathbb{R}_{+}^{S}$, and $i \in S$ it holds

$$
\mu\left(q_{-i}, C\right)=\sum_{\ell=1}^{k} \tilde{\alpha}_{\ell}\left\{C\left(\tilde{x}_{\ell}\right)-C\left(\tilde{x}_{\ell-1}\right)\right\} \quad \text { for all } c \in \mathcal{C}
$$

where $\tilde{x} \in \mathbb{R}^{k+1}$ and $\tilde{\alpha}_{1}, \tilde{\alpha}_{2}, \ldots, \tilde{\alpha}_{k} \in \Delta(S \backslash\{i\})$ are given for $\ell=1,2, \ldots, k$ by

$$
\tilde{x}_{\ell}=\sum_{1 \leq p \leq \ell}\left(1-\alpha_{p i}^{S}\right)\left(x_{p}^{S}-x_{p-1}^{S}\right)
$$




$$
\tilde{\alpha}_{\ell}= \begin{cases}\frac{\alpha_{\ell}^{S}}{\alpha_{\ell}^{S}(S \backslash\{i\})} & \text { if } \alpha_{\ell}^{S}(S \backslash\{i\})>0, \\ 0 & \text { else. }\end{cases}
$$

\subsection{Characterization of parametric cost sharing}

Note that, in the basic setup of our cost sharing model, except for the labeling of the agents, it is only their individual demand that may influence a solution, ceteris paribus. Then, if two agents can not be distinguished for these characteristics it is reasonable that they be treated equally by the solution.

Equal Treatment (ET) $q_{i}=q_{j} \Longrightarrow \mu_{i}(q, C)=\mu_{j}(q, C)$.

ET is implied by the anonymity property in Moulin and Shenker (1992). It can be used to refine Theorem 4.1, see Lemma 5.4 in the Appendix.

A rationing method $r$ is called continuous if it is jointly continuous in both arguments, i.e., $(q, t) \mapsto r(q, t)$ is continuous for all rationing problems $(q, t)$. Such $r$ is then robust against small changes in the parameters defining the rationing problem. For cost sharing mechanisms the approach is similar; a mechanism will be called continuous if small changes in demands and $B_{t}$ cause only small changes in cost shares. More specifically,

Continuity (CONT) The mapping $(q, t) \mapsto \mu\left(q, B_{t}\right)$ is continuous on $\mathbb{R}_{+}^{S} \times \mathbb{R}_{+} \cdot{ }^{10}$

Note that within the context of Theorem $4.1 \mu \in \mathcal{M}(\mathrm{ADD}, \mathrm{CR})$ is continuous if and only if the corresponding rationing method is. We will use this fact in order to characterize the cost sharing mechanisms related to parametric rationing methods that we will now define.

Consider a mapping $f: D \rightarrow \mathbb{R}$ be a real-valued function where $D \subset \mathbb{R}^{2}$ is a set in $\mathbb{R}_{+} \times[0, \Omega]$ for some $\Omega \in \mathbb{R}_{+} \cup\{\infty\}$. Assume that for $(z, \omega) \in D$ it holds that $f(z, 0)=0, f(z, \Omega)=z$ and $\omega \mapsto f(z, \omega)$ is non-decreasing and continuous. Then for such an $f$ there is a unique rationing method $r$ such that $r_{i}(q, t)=f\left(q_{i}, \omega\right)$ where $\omega$ solves $\sum_{i \in S} f\left(q_{i}, \omega\right)=t$. This $r$ is then called the parametric rationing method for $f$. See, e.g., Young (1987), Kaminski (2000), Moulin (2002) and Thomson (2003) for more examples and overviews. ${ }^{11}$ All earlier mentioned examples of consistent incremental mechanisms are parametric.

Proposition 4.7 $\mu \in \mathcal{M}(\mathrm{ADD}, \mathrm{CONS}, \mathrm{ET}, \mathrm{CONT})$ if and only if $\mu=\mu^{r}$ for some parametric $r$.

Proof If $r$ is parametric and $\mu=\mu^{r}$, then $\mu \in \mathcal{M}$ (ADD, CONS) by Theorem 4.2. Moreover, since any parametric method is continuous, by definition $\mu^{r}$ is continuous. Finally ET is implied by Lemma 5.4. The other way around follows just as in Young (1987), where the assumption of an infinite pool of agents is essential. In short, take $r$ with $\mu=\mu^{r}$ as in Theorem 4.1. Then in particular $r$ is continuous and Lemma 5.4

\footnotetext{
10 In order to avoid the hybrid character of CONT one may consider the replacement by two requirements, continuity of the mappings $t \mapsto \mu\left(q, B_{t}\right)$ and $q \mapsto \mu\left(q, B_{t}\right)$.

11 Moulin (2002) focuses on discrete formulation of the problem and asymmetric priority rules.
} 
shows that $r$ satisfies equal treatment. Now we are done by application of Theorem 1 in Young (1987).

\subsection{Consistent extensions}

Given a cost sharing mechanism defined for only 2-agent problems, it is natural to ask whether it is possible to extend it in a consistent way to a mechanism for arbitrarily finite sets of agents. The related problem for rationing methods has been partially solved. Dagan and Volij (1997) show that any monotonic rationing method for 2-agent problems allows for a unique average consistent method. And, moreover, whenever a consistent extension exists, it must coincide with the average consistent method. In particular this guarantees at most one consistent extension of a monotonic rationing method for 2-agent problems. Thomson (2008) shows the claims-truncated proportional rule as an example of a rationing method for 2-agent problems which cannot be extended consistently. Then from Theorem 4.2 we deduce:

Corollary 4.8 For each $\bar{\mu} \in \mathcal{M}(\mathrm{ADD}, \mathrm{CR})$ there is at most one $\mu \in \mathcal{M}$ (ADD, CONS) such that $\mu(P)=\bar{\mu}(P)$ for all $P \in \mathcal{P}^{S}$ with $|S|=2$.

For instance, this shows that there is at most one mechanism $\mu \in \mathcal{M}$ (ADD, CONS) extending serial- or average cost sharing for 2-agent problems, whereas there is no such extension of the mechanism defined through the truncated claims proportional method.

In principle, consistent cost sharing mechanisms can be constructed using the techniques of Dagan and Volij (1997). Once the rationing method underlying the mechanism for 2-agent problems is known, calculate the corresponding average consistent extension and-if at all-the consistent mechanism results from Theorem 4.2.

Acknowledgments I thank Hervé Moulin, William Thomson and Justin Leroux and the two anonymous referees for discussion and useful comments. The general idea for this paper was born at NPS, Monterey, and Rice University. Financial support by these institutes is gratefully acknowledged.

Open Access This article is distributed under the terms of the Creative Commons Attribution Noncommercial License which permits any noncommercial use, distribution, and reproduction in any medium, provided the original author(s) and source are credited.

\section{Appendix}

Lemma 5.1 Let $P=(q, C) \in \mathcal{P}^{S}$ and take $y \in \operatorname{CORE}^{*}(P)$. Then for each $i \in S$ there is $a \in[0, q(S \backslash\{i\})]$ such that $y_{i}=C_{P}^{*}\left(a+q_{i}\right)-C_{P}^{*}(a)$.

Proof Let $i \in S$ and define $g:[0, \infty) \rightarrow \mathbb{R}$ by $g(t)=C_{P}^{*}\left(t+q_{i}\right)-C_{P}^{*}(t)$. Then $g(0)=C_{P}^{*}\left(q_{i}\right) \geq y_{i}$ and $g(q(S \backslash\{i\}))=C_{P}^{*}(q(S))-C_{P}^{*}(q(S \backslash\{i\})) \leq y_{i}$. The latter inequality follows from the fact that $y \in \operatorname{CORE}^{*}(q, C)$ since $C_{P}^{*}(q(S))=y(S)$ and $C_{P}^{*}(q(S \backslash\{i\})) \geq y(S \backslash\{i\})$. Recall that $C_{P}^{*}$ is concave and thus continuous. Then by continuity of $g$ there exists $a$ such that $g(a)=y_{i}$. 
Lemma 5.2 (Tijs and Koster 1998) ${ }^{12}$ Let $P=(q, C) \in \mathcal{P}^{S}$. For each $x \in[0, q(S)]$ there is $T_{x} \subset[0, q(S)]$ such that $C_{P}^{*}(x)=\int_{T_{x}} C^{\prime}(t) \mathrm{d} t$ and $\lambda\left(T_{x}\right)=x$. The sets can be taken such that $x \leq y \Longrightarrow T_{x} \subseteq T_{y}$.

Lemma 5.3 For any cost sharing problem $P=(q, C) \in \mathcal{P}^{S}$ and each $y \in$ $\operatorname{CORE}^{*}(P)$ there exists a partition $\left\{U_{1}, U_{2}, \ldots, U_{|S|}\right\}$ of $[0, q(S)]$ such that for all $i \in S$ it holds that

$$
\begin{aligned}
& \text { (a) } \lambda\left(U_{i}\right)=q_{i}, \\
& \text { (b) } \int_{U_{i}} C^{\prime}(t) \mathrm{d} t=y_{i} .
\end{aligned}
$$

Proof First of all, we claim that for any $P=(q, C) \in \mathcal{P}^{S}, y \in \operatorname{CORE}^{*}(P)$ and $i \in S$ there is a $\lambda$-measurable set $T \subseteq[0, q(S)]$ with the following properties:

$$
\begin{aligned}
\text { (i) } & q_{i}=\lambda(T), \\
\text { (ii) } & \int_{T} C^{\prime}(t) \mathrm{d} t=y_{i}, \\
\text { (iii) } & y_{-i} \in \operatorname{CORE}^{*}\left(q_{-i}, C_{T}\right) .
\end{aligned}
$$

This is seen as follows. According to Lemma 5.1 there is an interval $\left[a, a+q_{i}\right] \subseteq$ $[0, q(S)]$ such that $y_{i}=C_{P}^{*}\left(a+q_{i}\right)-C_{P}^{*}(a)$. Consider a family of measurable sets $\left\{T_{z}\right\}_{z \in[0, q(S)]}$ as in Lemma 5.2 and define $T=T_{a+q_{i}} \backslash T_{a}$. Then $\int_{T} C^{\prime}(t) \mathrm{d} t=y_{i}$ and $\lambda(T)=q_{i}$. Let $\tilde{P}=\left(q_{-i}, C_{T}\right)$. Since $y \in \operatorname{CORE}^{*}(P)$ it holds for all $V \subseteq S \backslash\{i\}$ that $y(V \cup\{i\}) \leq C_{P}^{*}(q(V \cup\{i\}))$, so

$$
\begin{aligned}
y(V) & \leq C_{P}^{*}(q(V \cup\{i\}))-y_{i}= \\
& =\int_{T_{q(V \cup\{i\})}} C^{\prime}(t) \mathrm{d} t-\int_{T} C^{\prime}(t) \mathrm{d} t=\int_{T_{q(V \cup\{i\}) \backslash T}} C^{\prime}(t) \mathrm{d} t \\
& \leq \sup \left\{\int_{U} C^{\prime}(t) \mathrm{d} t \mid U \subseteq[0, q(S)] \backslash T, \lambda(U)=q(V)\right\} \\
& =\sup \left\{\int_{U}\left(C_{T}\right)^{\prime}(t) \mathrm{d} t \mid U \subseteq[0, q(S \backslash\{i\})], \lambda(U)=q(V)\right\} \\
& =\left(C_{T}\right)_{P}^{*}(q(V)) .
\end{aligned}
$$

By variation of $V$ and the fact that $y(S \backslash\{i\})=C_{T}(q(S \backslash\{i\}))$ we conclude that $y_{-i} \in \operatorname{CORE}^{*}(\tilde{P})$. This proves our claim. Then by repeated application of this claim we finish the proof.

12 Lemma 5.5, p. 156. 
Proof of Lemma 3.4 Coalitional consistency implies consistency, so we need only show that a consistent mechanism is coalitionally consistent. Let $P_{0}=\left(q, C_{0}\right)=$ $(q, C) \in \mathcal{P}^{S}, M=\left\{i_{1}, i_{2}, \ldots, i_{m}\right\} \subset S$. Define for $\ell \in\{1,2, \ldots, m\}, M_{\ell}=$ $\left\{i_{1}, i_{2}, \ldots, i_{\ell}\right\}$. Let $y=\mu(P)$, then by repeated application of consistency with respect to agents $i_{1}, i_{2}, \ldots, i_{m}$ we are assured of the existence of reduction mappings $\varphi^{1}, \varphi^{2}, \ldots, \varphi^{m}$ such that $\varphi^{\ell+1}$ is reduction mapping for $P_{\ell}=\left(q_{-M_{\ell}}, C_{\ell}\right) \in$ $\mathcal{P}^{S \backslash M_{\ell}}$ where $C_{\ell}=\left(C_{\ell-1}\right)_{\varphi^{\ell},-i_{\ell}}$, and $y_{-M_{\ell}}=\mu\left(P_{\ell}\right)$. So in particular we get $y=\mu(P) \Longrightarrow y_{-M}=\mu\left(q-M, C_{m}\right)$.

Now we are done by defining a reduction mapping $\varphi^{*}$ for $(\mu, P)$ such that $C_{\varphi^{*},-M}=$ $C_{m}$. Assuming that we have defined the coordinates $i_{1}, i_{2}, \ldots, i_{r-1}$ of $\varphi^{*}$, we denote $f_{r}^{*}:=\sum_{\ell<r} \varphi_{i_{\ell}}^{*}$ and $F_{r}^{*}:=\sum_{\ell<r} \Phi_{i_{\ell}}^{*}$, where $\Phi_{i_{\ell}}^{*}$ is the primitive of $\varphi_{i_{\ell}}^{*}$ such that $\Phi_{i_{\ell}}^{*}(0)=0$.

As a first step, define

$$
\varphi_{i_{1}}^{*}:=\varphi_{i_{1}}^{1}
$$

By virtue of $\varphi^{1}$ it holds that—as long as we see to it that $\varphi^{*}$ qualifies as reduction mapping - we have the following properties

$$
\left\{\begin{array}{l}
\int_{0}^{q(S)} \varphi_{i_{1}}^{*}(t) \mathrm{d} t=q_{i_{1}}, \\
\int_{0}^{q(S)} \varphi_{i_{1}}^{*}(t) C^{\prime}(t) \mathrm{d} t=\mu_{i_{1}}\left(P_{0}\right), \\
C_{\varphi^{*},-M_{1}}=C_{1} .
\end{array}\right.
$$

Suppose now that we have defined $\varphi_{i_{\ell}}^{*}$ for all $\ell \in\{1,2, \ldots, p-1\}$ for some $p \leq m$, so that

$$
\left\{\begin{array}{l}
\int_{0}^{q(S)} \varphi_{i_{\ell}}^{*}(t) \mathrm{d} t=q_{i_{\ell}}, \\
\int_{0}^{q(S)} \varphi_{i_{\ell}}^{*}(t) C^{\prime}(t) \mathrm{d} t=\mu_{i_{\ell}}\left(P_{\ell}\right)=\mu_{i_{\ell}}\left(P_{0}\right), \\
C_{\varphi^{*},-M_{\ell}}=C_{\ell} .
\end{array}\right.
$$

We will define $\varphi_{i_{p}}^{*}:[0, q(S)] \rightarrow[0,1]$ as follows

$$
\varphi_{i_{p}}^{*}(t)=\varphi_{i_{p}}^{p}\left(t-F_{p}^{*}(t)\right)\left(1-f_{p}^{*}(t)\right)
$$


Denote the primitive of $\varphi_{i_{p}}^{p}$ by $\Phi_{i_{p}}^{p}$. Use the substitution $u=t-F_{p}^{*}(t)$ and integrate to get the following property that is needed below

$$
\Phi_{i_{p}}^{*}(t)=\Phi_{i_{p}}^{p}\left(t-F_{p}^{*}(t)\right)
$$

First we will show that $\varphi^{*}$ is extended in an appropriate way. First of all

$$
\begin{aligned}
\int_{0}^{q(S)} \varphi_{i_{p}}^{*}(t) \mathrm{d} t & =\int_{0}^{q(S)} \varphi_{i_{p}}^{p}\left(t-F_{p}^{*}(t)\right)\left(1-f_{p}^{*}(t)\right) \mathrm{d} t \\
& =\int_{0}^{q\left(S \backslash M_{p-1}\right)} \varphi_{i_{p}}^{p}(y) \mathrm{d} y=q_{i_{p}},
\end{aligned}
$$

by virtue of $\varphi_{i_{p}}^{p}$. Implicit differentiation in (6) gives

$$
C_{\varphi^{*},-M_{p-1}}^{\prime}\left(t-F_{p}^{*}(t)\right)=C^{\prime}(t) \quad \text { whenever } 1-f_{p}^{*}(t) \neq 0 \text {. }
$$

Then

$$
\begin{aligned}
\int_{0}^{q(S)} \varphi_{i_{p}}^{*}(t) C^{\prime}(t) \mathrm{d} t=\int_{0}^{q(S)} \varphi_{i_{p}}^{p}\left(t-F_{p}^{*}(t)\right)\left(1-f_{p}^{*}(t)\right) C^{\prime}(t) \mathrm{d} t \\
\quad=\int_{0}^{q(S)} \varphi_{i_{p}}^{p}\left(t-F_{p}^{*}(t)\right)\left(1-f_{p}^{*}(t)\right) C_{\varphi^{*},-M_{p-1}}^{\prime}\left(t-F_{p}^{*}(t)\right) \mathrm{d} t \\
=\int_{0}^{q\left(S \backslash M_{p-1}\right)} \varphi_{i_{p}}^{p}(y) C_{\varphi^{*},-M_{p-1}}^{\prime}(y) \mathrm{d} t \\
=\mu_{i_{p}}\left(q_{-M_{p-1}}, C_{\varphi^{*},-M_{p-1}}\right)=\mu_{i_{p}}\left(q-M_{p-1}, C_{p-1}\right)=\mu_{i_{p}}(q, C),
\end{aligned}
$$

by the choice of $\varphi^{p}$, the fact that $C_{\varphi^{*},-M_{p-1}}=C_{p-1}$, and consistency.

Moreover

$$
\begin{aligned}
C_{\varphi^{*},-M_{p}}\left(y-F_{p+1}^{*}(y)\right) & =\int_{0}^{y}\left(1-f_{p+1}^{*}(t)\right) C^{\prime}(t) d t \\
& =\int_{0}^{y}\left(1-f_{p}^{*}(t)-\varphi_{i_{p}}^{*}(t)\right) C^{\prime}(t) d t
\end{aligned}
$$


Now use the definition of $\varphi_{i_{p}}^{*}$, the fact that $C^{\prime}(t)=C_{\varphi^{*},-M_{p-1}}^{\prime}\left(t-F_{p}^{*}(t)\right)$ whenever $1-f_{p}^{*}(t) \neq 0$ and rearranging of the terms to see that the above expression equals

$$
\int_{0}^{y}\left(1-\varphi_{i_{p}}^{p}\left(t-F_{p}^{*}(t)\right)\right)\left(1-f_{p}^{*}(t)\right) C_{\varphi^{*},-M_{p-1}}^{\prime}\left(t-F_{p}^{*}(t)\right) \mathrm{d} t
$$

By the substitution $u=t-F_{p}^{*}(t)$ and (13) expression (14) equals

$$
\begin{aligned}
& \int_{0}^{y-F_{p}^{*}(y)}\left(1-\varphi_{i_{p}}^{p}(u)\right) C_{\varphi^{*},-M_{p-1}}^{\prime}(u) \mathrm{d} u \\
& \quad=\left(C_{\varphi^{*},-M_{p-1}}\right)_{\varphi^{p},-i_{p}}\left(y-F_{p}^{*}(y)-\Phi_{i_{p}}^{p}\left(y-F_{p}^{*}(y)\right)\right) \\
& =\left(C_{\varphi^{*},-M_{p-1}}\right)_{\varphi^{p},-i_{p}}\left(y-F_{p}^{*}(y)-\Phi_{i_{p}}^{*}(y)\right) \\
& =\left(C_{\varphi^{*},-M_{p-1}}\right)_{\varphi^{p},-i_{p}}\left(y-F_{p+1}^{*}(y)\right) .
\end{aligned}
$$

Since $y \mapsto y-F_{p+1}^{*}(y)$ maps $[0, q(S)]$ onto $\left[0, q\left(S \backslash M_{p}\right)\right]$ we conclude that

$$
C_{\varphi^{*},-M_{p}} \equiv\left(C_{\varphi^{*},-M_{p-1}}\right)_{\varphi^{p},-i_{p}}=\left(C_{p-1}\right)_{\varphi^{p},-i_{p}}=C_{p}
$$

Then by inductive reasoning we may complete the definition of a reduction mapping $\varphi_{M}^{*}$ so that $C_{\varphi^{*},-M} \equiv C_{m}$. Complete $\varphi^{*}$ by taking for $\varphi_{S \backslash M}^{*}$ any available reduction mapping $\bar{\varphi}$ for $\left(\mu, P_{m}\right)$.

Proof of Lemma 3.5 Part 1: This is a direct consequence of Lemma 5.3.

Part 2: Take $P=(q, C) \in \mathcal{P}^{S}$ and let $y=\mu(P)$. If $\mu$ is consistent then there is a reduction map $\varphi$ for $(\mu, P)$ such that $y_{-i}=\mu\left(q_{-i}, C_{\varphi,-i}\right)$ for all $i \in S$. Take $V \subseteq S$, and define $X_{V}:=\left\{t \in[0, q(S)] \mid \sum_{i \in V} \varphi_{i}(t)>0\right\}$. Then $\lambda\left(X_{V}\right) \geq q(V)$ and

$$
\begin{aligned}
y(V) & =\sum_{i \in V} \int_{0}^{q(S)} \varphi_{i}(t) C^{\prime}(t) \mathrm{d} t=\int_{0}^{q(S)} \sum_{i \in V} \varphi_{i}(t) C^{\prime}(t) \mathrm{d} t \\
& \leq \sup _{U \subseteq X_{V}: \lambda(U)=q(V)} \int_{U} C^{\prime}(t) \mathrm{d} t \leq \sup _{U \subseteq[0, q(S)]: \lambda(U)=q(V)} \int_{U} C^{\prime}(t) \mathrm{d} t=C_{P}^{*}(q(V)) .
\end{aligned}
$$

But this means that $y$ is in the pessimistic core for $P$.

Proof of Theorem 4.2 Take $\mu \in \mathcal{M}$ (ADD, CONS). Recall that $\mu$ then satisfies CR, so that by Theorem $4.1 \mu=\mu^{r}$ for some monotonic rationing method $r$. Consider a cost sharing problem $P=\left(q, B_{x}\right)$ where $q \in \mathbb{R}_{+}^{S}, x \in[0, q(S)]$. Then $\mu\left(q, B_{x}\right)=r(q, x)$ by Theorem 4.1 . By consistency there is a reduction map $\varphi$ for $(\mu, P)$ such that 
$\mu_{-i}\left(q, B_{x}\right)=\mu\left(q_{-i},\left(B_{x}\right)_{\varphi,-i}\right)$ for all $i \in S$. Let $\Phi_{i}$ be the primitive of $\varphi_{i}$ with $\Phi_{i}(0)=0$. Then for $y \in[0, x]$

$$
\left(B_{x}\right)_{\varphi,-i}\left(y-\Phi_{i}(y)\right)=\int_{0}^{y}\left(1-\varphi_{i}(t)\right) B_{x}^{\prime}(t) \mathrm{d} t=\int_{0}^{y}\left(1-\varphi_{i}(t)\right) \mathrm{d} t=y-\Phi_{i}(y) .
$$

We may conclude that $\left(B_{x}\right)_{\varphi,-i}=B_{x-\Phi_{i}(x)}$. In particular, since $\varphi$ is a reduction map for $(\mu, P), \Phi_{i}(x)=\mu_{i}\left(q, B_{x}\right)=r_{i}(q, x)$, so

$$
r_{-i}(q, x)=\mu_{-i}\left(q, B_{x}\right)=\mu\left(q_{-i}, B_{x-r_{i}(q, x)}\right)=r\left(q_{-i}, x-r_{i}(q, x)\right)
$$

The other direction is straightforward. Take a consistent $r$ and let $\mu=\mu^{r}$. Define a reduction map $\varphi$ for $(\mu, P)$ as follows: $\varphi(t)=\frac{\partial}{\partial t} r(q, t)$ for all $t$ where it exists and let $\varphi(t)$ be an arbitrary unit vector otherwise. Take $i \in S$ and let $\Phi_{i}$ be as before, hence $\Phi_{i}(y)=r_{i}(q, y)$ for all $y .{ }^{13}$ Recall that $C_{\varphi,-i}\left(y-\Phi_{i}(y)\right)=\int_{0}^{y}\left(1-\varphi_{i}(t)\right) C^{\prime}(t) \mathrm{d} t$. Then by application of the chain rule we get

$$
\left(1-\varphi_{i}(y)\right)\left(C_{\varphi,-i}\right)^{\prime}\left(y-\Phi_{i}(y)\right)=\left(1-\varphi_{i}(y)\right) C^{\prime}(y) .
$$

So whenever $\varphi_{i}(y) \neq 1$ it holds $\left(C_{\varphi,-i}\right)^{\prime}\left(y-\Phi_{i}(y)\right)=C^{\prime}(y)$. This is just what we need, then

$$
\begin{aligned}
\mu_{-i}^{r}(q, C) & =\int_{0}^{q(S)} C^{\prime}(t) \mathrm{d} r_{-i}(q, t)=\int_{0}^{q(S)} C^{\prime}(t) \mathrm{d} r\left(q_{-i}, t-r_{i}(q, t)\right) \\
& =\int_{0}^{q(S)}\left(C_{\varphi,-i}\right)^{\prime}\left(t-\Phi_{i}(t)\right) \mathrm{d} r\left(q_{-i}, t-r_{i}(q, t)\right) \\
& =\int_{0}^{q(S)}\left(C_{\varphi,-i}\right)^{\prime}\left(t-\Phi_{i}(t)\right) \mathrm{d} r\left(q_{-i}, t-\Phi_{i}(t)\right) \\
& =\int_{0}^{q(S \backslash\{i\})}\left(C_{\varphi,-i}\right)^{\prime}(t) \mathrm{d} r(q-i, t)=\mu^{r}\left(q_{-i}, C_{\varphi,-i}\right) .
\end{aligned}
$$

\footnotetext{
13 Here I use the fact that a monotonic rationing method $r$ is Lipschitz-continuous and-in particularabsolutely continuous in the resource component $t$.
} 
Proof of Proposition 4.6 Suppose $\mu$ is consistent, then Theorem 4.2 implies that $\mu=$ $\mu^{r}$ where $r$ is the consistent and piecewise linear rationing method defined by

$$
r(q, t)=\sum_{h=1}^{\ell-1} \alpha_{h}^{S}\left(x_{h}^{S}-x_{h-1}^{S}\right)+\alpha_{\ell}^{S}\left(t-x_{\ell-1}^{S}\right) .
$$

So, by invoking consistency, it holds for $j \in S \backslash\{i\}$ and almost all $t \in\left[x_{\ell-1}^{S}, x_{\ell}^{S}\right]$ that

$$
\begin{aligned}
\alpha_{\ell j}^{S} & =\frac{\partial}{\partial t} r_{j}(q, t)=\frac{\partial}{\partial t} r_{j}\left(q_{-i}, t-r_{i}(q, t)\right) \\
& =\left.\frac{\partial}{\partial y} r_{j}\left(q_{-i}, y\right)\right|_{y=t-r_{i}(q, t)} \cdot \frac{\partial}{\partial t}\left(t-r_{i}(q, t)\right) \\
& =\left.\frac{\partial}{\partial y} r_{j}\left(q_{-i}, y\right)\right|_{y=t-r_{i}(q, t)} \cdot\left(1-\alpha_{\ell i}^{S}\right) \\
& =\left.\frac{\partial}{\partial y} r_{j}\left(q_{-i}, y\right)\right|_{y=t-r_{i}(q, t)} \cdot \alpha_{\ell}^{S}(S \backslash\{i\}) .
\end{aligned}
$$

Using that $\alpha_{\ell}^{S}(S \backslash\{i\})=0$ if and only if $x_{\ell-1}^{S}-r_{i}\left(q, x_{\ell-1}^{S}\right)=x_{\ell}^{S}-r_{i}\left(q, x_{\ell}^{S}\right)$, we conclude that on $\left(x_{\ell-1}^{S}-r_{i}\left(q, x_{\ell-1}^{S}\right), x_{\ell}^{S}-r_{i}\left(q, x_{\ell}^{S}\right)\right)$ the derivative of the mapping $y \mapsto r\left(q_{-i}, y\right)$ is constant. Now define $\bar{x}_{0}, \bar{x}_{1}, \ldots, \bar{x}_{k}$ and $\bar{\alpha}_{1}, \bar{\alpha}_{2}, \ldots, \bar{\alpha}_{k}$ by

$$
\begin{aligned}
& \bar{x}_{\ell}=x_{\ell}^{S}-r_{i}\left(q, x_{\ell}^{S}\right), \\
& \bar{\alpha}_{\ell}= \begin{cases}\alpha_{\ell}^{S} \cdot \alpha_{\ell}^{S}(S \backslash\{i\})^{-1} & \text { if } \alpha^{S}(S \backslash\{i\})>0, \\
0 & \text { else. }\end{cases}
\end{aligned}
$$

Then it holds that for all $t \in\left[\bar{x}_{\ell-1}, \bar{x}_{\ell}\right]$

$$
r\left(q_{-i}, t\right)=\sum_{h=1}^{\ell-1} \bar{\alpha}_{h}\left(\bar{x}_{h}-\bar{x}_{h-1}\right)+\bar{\alpha}_{\ell}\left(t-\bar{x}_{\ell-1}\right) .
$$

The definitions of $\bar{x}_{\ell}$ and $\bar{\alpha}_{\ell}$ match that of $\tilde{x}_{\ell}$ and $\tilde{\alpha}_{\ell}$ in (10) and (11), because

$$
\begin{aligned}
x_{\ell}^{S}-r_{i}\left(q, x_{\ell}^{S}\right) & =x_{\ell}^{S}-\sum_{h=1}^{\ell} \alpha_{h i}^{S}\left(x_{h}^{S}-x_{h-1}^{S}\right)=\sum_{h=1}^{\ell}\left(x_{h}^{S}-x_{h-1}^{S}\right)-\sum_{h=1}^{\ell} \alpha_{h i}^{S}\left(x_{h}^{S}-x_{h-1}^{S}\right) \\
& =\sum_{h=1}^{\ell}\left(1-\alpha_{h i}^{S}\right)\left(x_{h}^{S}-x_{h-1}^{S}\right) .
\end{aligned}
$$

Now we will prove the other way around. For all $\ell=1,2 \ldots, k$ we may choose a partition $\left(U_{\ell 1}, U_{\ell 2}, \ldots, U_{\ell|S|}\right)$ of $\left[x_{\ell-1}^{S}, x_{\ell}^{S}\right]$ such that (i) $\lambda\left(U_{\ell j}\right)=\alpha_{\ell j}^{S}\left(x_{\ell}^{S}-x_{\ell-1}^{S}\right)$ and (ii) $\int_{U_{\ell j}} C^{\prime}(t) \mathrm{d} t=\alpha_{\ell j}^{S}\left(C\left(x_{\ell}^{S}\right)-C\left(x_{\ell-1}^{S}\right)\right)$ for all $j \in S$. To see this, apply Lemma 5.3 
to each of the cost sharing problems $\left(\alpha^{S}\left(x_{\ell}^{S}-x_{\ell-1}^{S}\right), C_{\ell}\right)$ where $C_{\ell} \in \mathcal{C}$ is defined by $C_{\ell}(y):=C\left(y+\mathbf{x}_{\ell-1}^{S}\right)-C\left(x_{\ell-1}^{S}\right)$. Then $\varphi$ defined by $\varphi_{j}=\mathbb{I}_{U_{j}}$ for $U_{j}=\cup_{\ell=1}^{k} U_{\ell j}$ is a reduction map for $(\mu, P)$. Consider a reduction by agent $i$, then it is easily seen that (10) and (11) lead to the same cost shares for the agents in $S \backslash\{i\}$.

Proof of Theorem 4.5 In Example 3.3 strong consistency was shown for $\mu^{\mathrm{AV}}$, so we only have to show the uniqueness part. So suppose $\mu$ is an additive and strongly consistent cost sharing rule, not equal to $\mu^{\mathrm{AV}}$. We show that this leads to contradiction. Recall that $\mu$ satisfies constant returns. Consider a minimal set of agents $M$ for which there is $G=(d, C) \in \mathcal{P}^{M}$ with $d \in \mathbb{R}_{+}^{M}$ such that $\mu(G) \neq \mu^{\mathrm{AV}}(G)$. Let $i \in M$ be such that $\mu_{i}(G) \neq \mu_{i}^{\mathrm{AV}}(G)$. The agents share the costs, so there must as well be an agent $j \in M \backslash\{i\}$ with $\mu_{j}(G) \neq \mu_{j}^{\mathrm{AV}}(G)$. If it were the case that $M=N$, then in particular, by minimality of $M$, it holds for all $S \subsetneq N$ that

$$
G^{*} \in \mathcal{P}^{S} \Longrightarrow \mu\left(G^{*}\right)=\mu^{\mathrm{AV}}\left(G^{*}\right) .
$$

By Lemmas 3.5 and 5.3 we can choose a set $U_{i}$ so that $\mathbb{I}_{U_{i}}$ is a reducing map for $i$. In addition strong consistency implies $\mu_{j}\left(d_{-i}, C_{U_{i}}\right)=\mu_{j}^{\mathrm{AV}}\left(d_{-i}, C_{U_{i}}\right)$. For any such set $U_{i}$ we conclude, by (16) that

$$
\mu_{j}(d, c)=\mu_{j}\left(d_{-i}, C_{U_{i}}\right)=\mu_{j}^{\mathrm{Av}}\left(d_{-i}, C_{U_{i}}\right)=\mu_{j}^{\mathrm{AV}}(d, C),
$$

by strong consistency. But this contradicts with the choice of $j$, hence $M \subsetneq N$.

Now take $r$ with $\mu=\mu^{r}$ as in Theorem 4.1 and denote the derivative of $t \mapsto r(d, t)$ by $\hat{r}$. Then, in particular, $\lambda\left(\left\{y \in[0, d(M)] \mid \hat{r}_{i}(y) \neq \frac{d_{i}}{d(M)}\right\}\right)>0$. Define two measurable sets:

$$
T_{1}=\left\{s \in[0, d(M)] \mid \hat{r}_{i}(s)>\frac{d_{i}}{d(M)}\right\} \quad \text { and } \quad T_{0}=[0, d(M)] \backslash T_{1} .
$$

Note that $\lambda\left(T_{0}\right)>0$ and $\lambda\left(T_{1}\right)>0$. Choose another player in the nonempty set $N \backslash M$, say $k$. Define the demand profile $d^{*} \in \mathbb{R}_{+}^{M \cup\{k\}}$ such that $d_{M}^{*}=d$ and $d_{k}^{*}=d(M)$. Roughly, the proof proceeds by distinguishing several cases; for each case we define some cost sharing problem, that is reducible with respect to agent $k$ and such that cost shares in those reductions depend on the reduction. Then, as a result, a contradiction with strong consistency remains.

First, consider the cost function $C$ defined by

$$
C(t)=\int_{0}^{t} \mathbb{I}_{A}(s) \mathrm{d} s,
$$

where $A=T_{0} \cup\left[d(M), d(M)+\lambda\left(T_{1}\right)\right]$. Distinguish three cases:

Case 1: $\mu_{k}\left(d^{*}, C\right)=0$

Case 2: $\mu_{k}\left(d^{*}, C\right)=d(M)$

Case 3: $0<\mu_{k}\left(d^{*}, C\right)<d(M)$ 
Case 1: $\mu_{k}\left(d^{*}, C\right)=0$. For notational convenience use $\hat{r}^{*}$ to denote the derivative of $t \mapsto r\left(d^{*}, t\right)$. Then

$$
\hat{r}_{k}^{*}=\left\{\begin{array}{l}
1 \text { a.e. on } T_{1} \cup\left[2 d(M)-\lambda\left(T_{0}\right), 2 d(M)\right], \\
0 \text { a.e. on } T_{0} \cup\left[d(M), d(M)+\lambda\left(T_{1}\right)\right] .
\end{array}\right.
$$

Again we distinguish between several cases:

(a) Almost all first $\lambda\left(T_{0}\right)$ levels are in $T_{0}$.

(b) Almost all first $\lambda\left(T_{1}\right)$ levels are in $T_{1}$.

(c) Not case $(a)$, nor $(b)$.

- Case $1(a)$ and $(b)$ : Consider the cost function $\bar{C}$ defined by

$$
\bar{C}(y)=\int_{0}^{y} \mathbb{I}_{A}(s) \mathrm{d} s
$$

where $A=T_{0} \cup\left[2 d(M)-\lambda\left(T_{0}\right), 2 d(M)\right]$. Then $\mu_{k}\left(d^{*}, \bar{C}\right)=\lambda\left(T_{0}\right)$ by (18). Now it is possible to reduce the cost sharing problem $\left(d^{*}, \bar{C}\right)$ by agent $k$ in two ways, such that the corresponding cost shares of agent $i$ differ. To see this consider the two reductions $\left(d, \bar{C}_{U}\right)$ with $U=[0, d(M)]$, and $\left(d, \bar{C}_{W}\right)$ with $W=$ $[d(M), 2 d(M)]$.

In Case 1(a), if almost all first $\lambda\left(T_{0}\right)$ levels are in $T_{0}$, then $\lambda\left(\left[0, \lambda\left(T_{0}\right)\right] \cap T_{0}\right)=$ $\lambda\left(T_{0}\right)$.

(i) If $\lambda\left(T_{1}\right) \leq \lambda\left(T_{0}\right)$, then

$$
\begin{aligned}
\mu_{i}\left(d, \bar{C}_{U}\right)-\mu_{i}\left(d, \bar{C}_{W}\right)= & \int_{T_{1}} \hat{r}_{i}(s) \mathrm{d} s-\int_{0}^{\lambda\left(T_{1}\right)} \hat{r}_{i}(s) \mathrm{d} s \\
& >\lambda\left(T_{1}\right) \cdot \frac{d_{i}}{d(M)}-\lambda\left(T_{1}\right) \cdot \frac{d_{i}}{d(M)}=0 .
\end{aligned}
$$

(ii) If $\lambda\left(T_{1}\right)>\lambda\left(T_{0}\right)$, then

$$
\begin{aligned}
\mu_{i}\left(d, \bar{C}_{U}\right)-\mu_{i}\left(d, \bar{C}_{W}\right)= & \int_{\lambda\left(T_{1}\right)}^{d(M)} \hat{r}_{i}(s) \mathrm{d} s-\int_{0}^{\lambda\left(T_{0}\right)} \hat{r}_{i}(s) \mathrm{d} s \\
& >\lambda\left(T_{0}\right) \cdot \frac{d_{i}}{d(M)}-\lambda\left(T_{0}\right) \cdot \frac{d_{i}}{d(M)}=0 .
\end{aligned}
$$

Or, equivalently (combining (i) and (ii)),

$$
\begin{aligned}
\mu_{i}\left(d, \bar{C}_{U}\right)-\mu_{i}\left(d, \bar{C}_{W}\right)= & \int_{T_{1}} \hat{r}_{i}(s) \mathrm{d} s-\int_{0}^{\min \left\{\lambda\left(T_{1}\right), \lambda\left(T_{0}\right)\right\}} \hat{r}_{i}(s) \mathrm{d} s> \\
& >\lambda\left(T_{1}\right) \cdot \frac{d_{i}}{d(M)}-\min \left\{\lambda\left(T_{0}\right), \lambda\left(T_{1}\right)\right\} \cdot \frac{d_{i}}{d(M)} \geq 0 .
\end{aligned}
$$


This contradicts consistency.

In Case 1(b), if almost all first $\lambda\left(T_{1}\right)$ levels are in $T_{1}$, or equivalently $\lambda\left(\left[0, \lambda\left(T_{1}\right)\right] \cap T_{1}\right)=\lambda\left(T_{1}\right)$, then

$$
\begin{aligned}
& \mu_{i}\left(d, \bar{C}_{U}\right)-\mu_{i}\left(d, \bar{C}_{W}\right) \\
& =\int_{0}^{\min \left\{\lambda\left(T_{0}\right), \lambda\left(T_{1}\right)\right\}} \hat{r}_{i}(s) \mathrm{d} s-\int_{d(M)-\min \left\{\lambda\left(T_{0}\right), \lambda\left(T_{1}\right)\right\}}^{d(M)} \hat{r}_{i}(s) \mathrm{d} s \\
& \quad>\min \left\{\lambda\left(T_{0}\right), \lambda\left(T_{1}\right)\right\} \cdot \frac{d_{i}}{d(M)}-\int_{d(M)-\min \left\{\lambda\left(T_{0}\right), \lambda\left(T_{1}\right)\right\}}^{d(M)} \hat{r}_{i}(s) \mathrm{d} s \geq \\
& \geq \min \left\{\lambda\left(T_{0}\right), \lambda\left(T_{1}\right)\right\} \cdot \frac{d_{i}}{d(M)}-\min \left\{\lambda\left(T_{0}\right), \lambda\left(T_{1}\right)\right\} \cdot \frac{d_{i}}{d(M)}=0 .
\end{aligned}
$$

This contradicts consistency.

- Case $1(c)$ : The first levels are not all of type $T_{0}$ nor of type $T_{1}$, in $\lambda$-sense. Consider the cost sharing problem $\left(d^{*}, \tilde{C}\right)$, with

$$
\tilde{C}(y)=\int_{0}^{y} \mathbb{I}_{A}(s) \mathrm{d} s, A=T_{1} \cup\left[d(M), d(M)+\lambda\left(T_{1}\right)\right] .
$$

Then $\mu\left(d^{*}, \tilde{C}\right)=\lambda\left(T_{1}\right)$ by (18). Look at the reductions by agent $k,\left(d, \tilde{C}_{U}\right)$ and $\left(d, \tilde{C}_{W}\right):$ with $U=[0, d(M)], W=[d(M), 2 d(M)]$. Then

$$
\mu_{i}\left(d, \tilde{C}_{U}\right)-\mu_{i}\left(d, \tilde{C}_{W}\right)=\int_{0}^{\lambda\left(T_{1}\right)} \hat{r}_{i}(s) \mathrm{d} s-\int_{T_{1}} \hat{r}_{i}(s) \mathrm{d} s<0,
$$

contradicting consistency.

Case 2: $\mu_{k}\left(d^{*}, C\right)=d(M)$. Then

$$
\hat{r}_{k}^{*}=\left\{\begin{array}{l}
0 \text { a.e. on } T_{1} \cup\left[2 d(M)-\lambda\left(T_{0}\right), 2 d(M)\right], \\
1 \text { a.e. on } T_{0} \cup\left[d(M), d(M)+\lambda\left(T_{1}\right)\right] .
\end{array}\right.
$$

Distinguish the following two cases

(a) Almost all first $\lambda\left(T_{1}\right)$ levels are in $T_{1}$.

(b) Not all first $\lambda\left(T_{1}\right)$ levels are in $T_{1}$.

- Case $2(a): \lambda\left(T_{1} \cap\left[0, \lambda\left(T_{1}\right)\right]\right)=\lambda\left(T_{1}\right)$. Consider the cost function $\bar{C}$ as previously defined in (19). It holds that $\mu_{k}\left(d^{*}, \bar{C}\right)=\lambda\left(T_{0}\right)$ by (20). Then reductions by agent $k$ are defined by $\left(d, \bar{C}_{U}\right)$ with $U=\left[0, \lambda\left(T_{1}\right)\right] \cup\left[d(M)+\lambda\left(T_{1}\right), 2 d(M)\right]$, 
and $\left(d, \bar{C}_{W}\right)$ with $W=[d(M), 2 d(M)]$. Then

$$
\begin{aligned}
\mu_{i}\left(d, \bar{C}_{U}\right)-\mu_{i}\left(d, \bar{C}_{W}\right)= & \int_{0}^{\min \left\{\lambda\left(T_{0}\right), \lambda\left(T_{1}\right)\right\}} \hat{r}_{i}(s) \mathrm{d} s \\
& -\int_{d(M)-\min \left\{\lambda\left(T_{0}\right), \lambda\left(T_{1}\right)\right\}}^{d(M)} \hat{r}_{i}(s) \mathrm{d} s>0,
\end{aligned}
$$

contradicting consistency.

- Case $2(b)$ : Suppose that not almost all first $\lambda\left(T_{1}\right)$ production levels are in $T_{1}$. Take $\epsilon \in\left(0, \lambda\left(T_{1}\right)\right)$ and define the cost function

$$
\tilde{C}(y)=\int_{0}^{y} \mathbb{I}_{A}(s) \mathrm{d} s, \quad \text { where } A=T_{1} \cup\left[d(M)+\lambda\left(T_{1}\right)-\epsilon, d(M)+\lambda\left(T_{1}\right)\right] .
$$

Then by (20) it holds that $\mu_{i}\left(d^{*}, \tilde{C}\right)=\epsilon$. Now consider the following two reductions by agent $k,\left(d, \tilde{C}_{U}\right)$ and $\left(d, \tilde{C}_{W}\right)$, where $U=[d(M), 2 d(M)]$ and $W=T_{0} \cup\left[d(M), d(M)+\lambda\left(T_{1}\right)\right]$. Then

$$
\mu_{i}\left(d, \tilde{C}_{U}\right)-\mu_{i}\left(d, \tilde{C}_{W}\right)=\int_{T_{1}} \hat{r}_{i}(s) \mathrm{d} s-\int_{0}^{\lambda\left(T_{1}\right)} \hat{r}_{i}(s) \mathrm{d} s>0
$$

contradicting strong consistency.

Case 3: $0<\mu_{k}\left(d^{*}, C\right)<d(M)$. Distinguish two cases

(a) $\int_{T_{0}} \hat{r}_{k}^{*}(s) \mathrm{d} s>0$,

(b) $\int_{T_{0}} \hat{r}_{k}^{*}(s) \mathrm{d} s=0$.

- Case $3(a): \int_{T_{0}} \hat{r}_{k}^{*}(d) \mathrm{d} s>0$. For $\alpha \in[0, d(M)]$ define the cost function $C^{\alpha}$ by

$$
C^{\alpha}(y)=\int_{0}^{y} \mathbb{I}_{A}(s) \mathrm{d} s, \text { where } A=T_{0} \cup[d(M), d(M)+\alpha]
$$

Then the function $f: \alpha \mapsto \mu_{k}\left(d^{*}, C^{\alpha}\right)-\alpha$ is continuous on $[0, d(M)]$. Moreover

$$
\begin{aligned}
& f(0)=\mu_{k}\left(d^{*}, C^{0}\right)-0=\int_{T_{0}} \hat{r}_{k}^{*}(s) \mathrm{d} s>0, \text { and } \\
& f(d(M))=\mu_{k}\left(d^{*}, C^{d(M)}\right)-d(M) \leq 0 .
\end{aligned}
$$

So there is $\beta \in(0, d(M)]$ such that $f(\beta)=0$, which means that $\mu_{k}\left(d^{*}, C^{\beta}\right)=\beta$. 
(a.1) First, assume that almost all $T_{1}$ levels are at the end of the interval $[0, d(M)]$, which means that $\lambda\left(T_{1} \cap\left[\lambda\left(T_{0}\right), d(M)\right]\right)=\lambda\left(T_{1}\right)$. Then one reduction by agent $k$ is defined by $\left(d, C_{W}^{\beta}\right)$ for $W=[d(M), 2 d(M)]$. Another is $\left(d, C_{U}^{\beta}\right)$ for $U=[0, t] \cup[d(M)+t, 2 d(M)]$ and $t=\min \left\{\lambda\left(T_{0}\right), \lambda\left(T_{1}\right), \beta\right\}$. By definition of $t$ it holds $\lambda\left([d(M)-t, d(M)] \cap T_{0}\right)=0$, i.e. almost all production levels in the interval $[d(M)-t, d(M)]$ are in $T_{1}$. Then notice that $C_{W}^{\beta}(y)=$ $\int_{0}^{y} \mathbb{I}_{T_{0}}(s) \mathrm{d} s$ to conclude that

$$
\mu_{i}\left(d, C_{U}^{\beta}\right)-\mu_{i}\left(d, C_{W}^{\beta}\right)>0
$$

contradicting strong consistency.

(a.2) Assume not almost all first $\lambda\left(T_{1}\right)$ levels are in $T_{1}$, or $\lambda\left(T_{1} \cap\left[0, \lambda\left(T_{0}\right)\right]\right)<$ $\lambda\left(T_{1}\right)$. Then there exists $y \in\left(0, \lambda\left(T_{1}\right)\right)$ such that $\lambda\left([0, y] \cap T_{0}\right)>0$. Write $Y=[0, y] \cap T_{0}$. Note that $\mu_{k}\left(d^{*}, C^{\beta}\right)=\beta$. Consider the reduction by agent $k$, $\left(d, C_{U}^{\beta}\right)$ and $\left(d, C_{W}^{\beta}\right)$ with $U=Y \cup[d(M)+\lambda(Y), 2 d(M)]$ and $W=[d(M)$, $2 d(M)]$, respectively. Then $\mu_{i}\left(d, C_{W}^{\beta}\right)-\mu_{i}\left(d, C_{U}^{\beta}\right)>0$, contradicting strong consistency.

Case 3 (b): Suppose that $\int_{T_{0}} \hat{r}_{k}^{*}(s) \mathrm{d} s=0$. Then in particular $\mu_{k}\left(d^{*}, C\right) \leq \lambda\left(T_{1}\right)$. Then there are two cases:

(b.1) $\mu_{i}\left(d^{*}, C\right)=\lambda\left(T_{1}\right)$

(b.2) $\mu_{i}\left(d^{*}, C\right)<\lambda\left(T_{1}\right)$

(b.1) Suppose that $\mu_{k}\left(d^{*}, C\right)=\lambda\left(T_{1}\right)$. Then obviously one reduction by agent $k$ is specified by $\left(d, C_{A}\right)$ with $A=[d(M), 2 d(M)]$. Define $q=\min \left\{\lambda\left(T_{0}\right), \lambda\left(T_{1}\right)\right\}$. Assume that all first $\lambda\left(T_{0}\right)$ production levels are in $T_{0}$, i.e. $\lambda\left(\left[0, \lambda\left(T_{0}\right)\right] \cap T_{0}\right)=$ $\lambda\left(T_{0}\right)$. Then $\left(d, C_{B}\right)$ with $B=[0, q] \cup[d(M)+q, 2 d(M)]$ defines another reduction by agent $k$. Observe that in this reduced cost function all levels in $[d(M)-q, d(M)]$ have marginal costs 1 , which are almost all contained in $T_{1}$. So $\mu_{i}\left(d, C_{A}\right)<\mu_{i}\left(d, C_{B}\right)$, contradicting SCONS. Hence, assume that not almost all first $\lambda\left(T_{0}\right)$ production levels are in $T_{0}$. Then there exists $y \in\left(0, \lambda\left(T_{0}\right)\right)$ such that $\lambda\left([0, y] \cap T_{1}\right)>0$. Let $Y=[0, y] \cap T_{1}$ and $U=Y \cup[d(M)+\lambda(Y), 2 d(M)]$. Then $\left(d, C_{U}\right)$ constitutes another reduction by agent $k$ such that-similar to Case 3 (a.2)- $\mu_{i}\left(d, C_{U}\right)>\mu_{i}\left(d, C_{A}\right)$, contradiction.

(b.2) Assume that $\mu_{k}\left(d^{*}, C\right)<\lambda\left(T_{1}\right)$. Then $\lambda\left(\left\{s \in T_{1} \mid \hat{r}_{k}^{*}(s)=0\right\}\right)>0$ would imply $\mu_{k}\left(d^{*}, C\right)=\lambda\left(T_{1}\right)$, contradiction. So $\lambda\left(\left\{s \in T_{1} \mid \hat{r}_{k}^{*}(s)>0\right\}\right)>0$, and $\mu_{k}\left(d^{*}, \bar{C}\right)>0$, where $\bar{C}$ is the cost function defined by

$$
\bar{C}(y)=\int_{0}^{y} \mathbb{I}_{T_{1}}(s) \mathrm{d} s .
$$


Then it holds $0<\mu_{k}\left(d^{*}, \bar{C}\right) \leq \lambda\left(T_{1}\right)$. Suppose that almost all $T_{0}$ production levels are at the beginning. Define sets

$$
\begin{aligned}
U & =\left[0, \lambda\left(T_{0}\right)+\mu_{k}\left(d^{*}, \bar{C}\right)\right] \cup\left[d(M), d(M)+\lambda\left(T_{1}\right)-\mu_{k}\left(d^{*}, \bar{C}\right)\right] \\
V & =\left[d(M)-\mu_{k}\left(d^{*}, \bar{C}\right), 2 d(M)-\mu_{k}\left(d^{*}, \bar{C}\right)\right] .
\end{aligned}
$$

Then $\left(d, \bar{C}_{U}\right)$ and $\left(d, \bar{C}_{V}\right)$ are reductions from $\left(d^{*}, \bar{C}\right)$ by agent $k$. Note that in the second reduction, almost all $\lambda\left(T_{1}\right)-\mu_{k}\left(d^{*}, \bar{C}\right)$ levels with marginal cost 1 are in $T_{1}$. So this means that here agent $i$ is strictly worse off than in the first case where $\lambda\left(T_{1}\right)-\mu_{k}\left(d^{*}, C\right)$ marginal cost levels 1 are in $T_{0}$, or $\mu_{i}\left(d, \bar{C}_{U}\right)<\mu_{i}\left(d, \bar{C}_{V}\right)-$ contradiction. So it can not happen that almost all first productions levels are in $T_{0}$. Then, analogously to Case 3 (a.1), define the cost function $\bar{C}^{\alpha}$

$$
\bar{C}^{\alpha}(y)=\int_{0}^{y} \mathbb{I}_{A}(s) \mathrm{d} s, \quad \text { with } A=T_{1} \cup[d(M)-\alpha, d(M)], \text { and }
$$

consider the function $f: \alpha \mapsto \mu_{k}\left(d^{*}, \bar{C}^{\alpha}\right)-\alpha$. Then $f$ is continuous and

$$
\begin{aligned}
& f(0)=\mu_{k}\left(d^{*}, \bar{C}^{0}\right)=\mu_{k}\left(d^{*}, \bar{C}\right)>0 \\
& f(d(M))=\mu_{k}\left(d^{*}, \bar{C}^{d(M)}\right)-d(M)<0 .
\end{aligned}
$$

So, there is $\beta \in(0, d(M))$ such that $\mu_{k}\left(d^{*}, \bar{C}^{\beta}\right)=\beta$. If almost all first $\lambda\left(T_{1}\right)$ production levels are in $T_{1}$ then consider the reductions $\left(d, \bar{C}_{U}^{\beta}\right)$ by agent $k$ and $\left(d, \bar{C}_{W}^{\beta}\right)$ with $U=[d(M)-\beta, 2 d(M)-\beta], W=\left[\lambda\left(T_{1}\right)-\epsilon, \lambda\left(T_{1}\right)\right] \cup[d(M), 2 d(M)-\epsilon]$ and where $\epsilon \in\left(0, \min \left\{\lambda\left(T_{1}\right), \beta\right\}\right)$. Then in the first reduction almost all marginal cost levels 1 are in $T_{1}$. In the second reduction there are $\epsilon$ less in $T_{1}$ and $\epsilon$ more in $T_{0}$. Then this means that $\mu_{i}\left(d, \bar{C}_{U}^{\beta}\right)>\mu_{i}\left(d, \bar{C}_{W}^{\beta}\right)$, contradiction. The case where not almost all first $\lambda\left(T_{1}\right)$ production levels are in $T_{1}$ is treated analogously to Case 3 (a.2): one reduction by agent $k$ is $\left(d, \bar{C}_{U}^{\beta}\right)$ whereas another can be found with a cost function with less marginal cost levels 1 in $T_{1}$.

\section{Lemma $5.4 \mu \in \mathcal{M}(\mathrm{ADD}, \mathrm{CR}, \mathrm{ET})$ if and only if}

(i) $\mu$ is generated by a rationing method $r$, i.e., $\mu=\mu^{r}$,

(ii) iffor $q \in \mathbb{R}_{+}^{S}, q_{i}=q_{j}$ for some $i, j \in S$, then $r_{i}(q, \cdot)=r_{j}(q, \cdot)$.

Proof The combination of properties ADD, CR implies the functional representation as in Theorem 4.1. Now suppose there is $q \in \mathbb{R}_{+}^{S}$ and $i, j \in S$ with $q_{i}=q_{j}$ but not $r_{i}(q, \cdot)=r_{j}(q, \cdot)$, and an interval $U \subset[0, q(S)]$ such that $\mathrm{d} r_{i}(q, \cdot)<\mathrm{d} r_{j}(q, \cdot)$ on $U$. Consider $C \in \mathcal{C}$ defined by $C(y)=\int_{0}^{y} \mathbb{I}_{U}(t) \mathrm{d} t$ for all $y \in \mathbb{R}_{+}$. Then $\mu_{i}(q, C)=$ $\int_{0}^{q(S)} \mathbb{I}_{U}(t) \mathrm{d} r_{i}(q, t)<\int_{0}^{q(S)} \mathbb{I}_{U}(t) \mathrm{d} r_{j}(q, t)=\mu_{j}(q, C)$, which gives the desired contradiction. 
Open Access This article is distributed under the terms of the Creative Commons Attribution Noncommercial License which permits any noncommercial use, distribution, and reproduction in any medium, provided the original author(s) and source are credited.

\section{References}

Albizuri MJ, Zarzuelo JM (2007) The dual serial cost-sharing rule. Math Soc Sci 53:150-163

Dagan N, Volij O (1997) Bilateral comparisons and consistent fair division rules in the context of bankruptcy problems. Int J Game Theory 26:11-25

Davis M, Maschler M (1965) The kernel of a cooperative game. Naval Res Logist Q 12:223-259

Hardy GH, Littlewood JE, Pólya G (1959) Inequalities. 2nd ed. Cambridge University Press, London

Hart S, Mas-Colell A (1989) Potential, value and consistency. Econometrica 57:589-614

Hougaard JL, Thorlund-Petersen L (2001) Mixed serial cost sharing. Math Soc Sci 41:51-68

Kaminski M (2000) 'Hydrolic' rationing. Math Soc Sci 40:131-155

Kolpin V (1994) Coalition-equitable cost sharing of multi-service facilities. Mimeo, University of Oregon

Koster M (2002) Concave and convex serial cost sharing. In: Borm P, Peters H (eds) Chapters in game theory. Kluwer, Dordrecht

Koster M (2009) Contracts, cost sharing and consistency. CeNDEF Working paper 09-04, University of Amsterdam, The Netherlands

Koster M (2009) Cost sharing. In: Meyers RA (ed) Encyclopedia of complexity and systems science. Springer, Berlin pp 1660-1689

Lebesgue H (1904) Leçons sur l'intégration et la recherche des fonctions primitives. Gauthier-Villars, Paris Leroux J (2007) The consistency axiom in cost-allocation problems: a discussion. Mimeo

Maschler M (1990) Consistency. In: Ichiishi T, Neyman A, Tauman Y (eds) Game theory and applications. Academic Press, New York pp 183-186

Moulin H (2000) Priority rules and other asymmetric rationing methods. Econometrica 68:643-684

Moulin H (2002) Axiomatic cost and surplus-sharing. In: Arrow KJ, Sen AK, Suzumura K (eds) Handbook of social choice and welfare. Handbooks in economics. North-Holland, Amsterdam pp 289-357

Moulin H, Shenker S (1992) Serial cost sharing. Econometrica 60:1009-1037

Moulin H, Shenker S (1994) Average cost pricing versus serial cost sharing; an axiomatic comparison. J Econ Theory 64:178-201

Ryff JV (1970) Measure preserving transformation and rearrangements. J Math Anal Appl 31:449-458

Sharkey W (1982) The theory of natural monopoly. Cambridge University Press, Cambridge

Shubik M (1962) Incentives, decentralized control, the assignment of joint cost, and internal pricing. Manag Sci 8:325-343

Sobolev AI (1973) The functional equations that give the payoffs of the players in an $n$-person game. In: Vilkas E (ed) Advances in game theory. Izdat. "Mintis", Vilnius, pp 151-153

Sudhölter P (1998) Axiomatizations of game theoretical solutions for one-output cost sharing problems. Games Econ Behav 24:42-71

Thomson W (2001) On the axiomatic method and its recent applications to game theory and resource allocation. Soc Choice Welf 18:327-386

Thomson W (2003) Axiomatic and game-theoretic analysis of bankruptcy and taxation problems: a survey. Math Soc Sci 45:249-297

Thomson W (2006) Consistent allocation rules. Mimeo, Economics Department, University of Rochester, Rochester

Thomson W (2008) The two-agent claims-truncated proportional rule has no consistent extension: a constructive proof. Econ Lett 98:59-65

Tijs SH, Koster M (1998) General aggregation of demand and cost sharing methods. Ann Oper Res 84: 137-164

Weber RJ (1988) Probabilistic values for games. In: Roth AE (ed) The Shapley value. Cambridge University Press, Cambridge

Young HP (1985) Cost allocation: methods, principles, applications. North-Holland, Amsterdam

Young HP (1987) On dividing an amount according to individual claims or liabilities. Math Oper Res 12:398-414

Young HP (1994) Cost allocation. In: Aumann RJ, Hart S (eds) Handbook of game theory II. Elsevier, Amsterdam pp 1193-1235 